\title{
On the validity of "A proof that the discrete singular convolution (DSC)/Lagrange-distributed approximation function (LDAF) method is inferior to high order finite differences"
}

\author{
G. W. Wei ${ }^{1,2 *}$ and Shan Zhao ${ }^{1}$ \\ ${ }^{1}$ Department of Mathematics, \\ Michigan State University, East Lansing, MI 48824, USA \\ ${ }^{2}$ Department of Electrical and Computer Engineering, \\ Michigan State University, East Lansing, MI 48824, USA
}

November 9, 2018

\begin{abstract}
A few families of counterexamples are provided to "A proof that the discrete singular convolution (DSC)/Lagrange-distributed approximation function (LDAF) method is inferior to high order finite differences", Journal of Computational Physics, 214, 538-549 (2006).
\end{abstract}

\section{Introduction}

Recently, Boyd published a paper entitled "A proof that the discrete singular convolution (DSC)/Lagrangedistributed approximating function (LDAF) method is inferior to high order finite differences" [] which will be referred to as "Proof" throughout the present work. The sole purpose of the present paper is to analyze the validity of many of the statement and claims in "Proof". To this end, we provide a wide variety of counterexamples. It is pointed out that we have no intention to claim the superiority of the DSC algorithm. In the rest of the Introduction, we shall set the scope of our discussions.

${ }^{*}$ Corresponding author. Tel: (517)3534689, Fax: (517)4321562, Email: wei@math.msu.edu 
For the DSC algorithm, only a special DSC kernel will be discussed. "Proof" makes very general claims about the DSC algorithm. Unfortunately, it ignores the fact that the DSC algorithm can be realized by many different kernels that might behave very differently from each other [22]. What is discussed in "Proof" is a special DSC kernel, the regularized Shannon kernel (RSK). We therefore limit our discussion to the DSC-RSK method exclusively in the present work.

One of "Proof"'s central claims is about spectrally-weighted differences. "Proof" states that "Although we shall not perform detailed comparisons between DSC and spectrally-weighted differences, the good performance of DSC for high $k$ and small $a$ is an accident. It seems likely that for $f(x)$ which are known to have spectra concentrated between $K=\pi / 2$ and $K=\pi$, one could obtain higher accuracy from spectrally-weighted differences than from DSC". It is seen that "Proof" does not provide any detailed comparisons between the DSC and spectrally-weighted differences. Therefore, "Proof" has to present its claim about the superiority of the spectrally-weighted differences over the DSC as a shaky speculation: "It seems likely that ...". Unfortunately, this unfounded superiority was a part of "Proof"'s main claims. Although there was no proof to begin with, "Proof" still concludes that "DSC/LDAF methods are never the best way to approximate derivatives on a stencil of a given width" in its abstract and "the DSC/LDAF method is never the method of choice for approximating derivatives" in its summary. In this work, we will analyze the validity of this claim in detail. Since "Proof"'s claim about spectrally-weighted differences is very general, logically, it is sufficient for us to discredit this general claim by considering a special case. To this end, we choose Boyd's sech function based spectrally-weighted difference (Sech) [5], for which a construction procedure was emphasized and outlined in "Proof". It is pointed out that our results in this work carry no implication on the performance of any other spectrally-weighted differences.

The other part of "Proof"'s central claims is about the standard high order finite difference (FD) scheme. "Proof" classifies functions into two classes, namely, those with small (Fourier) wavenumbers and those with large (Fourier) wavenumbers, and then argues that the standard high order finite difference (FD) scheme is more accurate than the DSC in differentiating functions with considerable amplitude in small wavenumbers, while spectrally-weighted differences, e.g., the Sech method, are superior for functions with (solely) large wavenumbers. "Proof" claims that "Consequently, DSC/LDAF methods are never the best way to approximate derivatives on a stencil of given width".

It should be pointed out that no part of "Proof"'s claims is based on rigorous error analysis. 
Error analysis for the DSC-RSK method was given earlier by Qian [19], and was not mentioned in "Proof". Although error expressions for both the FD and DSC-RSK methods are presented, these expressions are not compared and do not directly support "Proof"'s claims. Instead, "Proof"'s claim about the superiority of the FD method over the DSC-RSK method is based on numerical experiments with a limited set of parameters, and on some informal arguments. In this work, we present a counterexample in differentiating $e^{i k x}$ with a small wavenmuber $k$, see Fig. 1, In differentiating a function with exponentially decaying amplitudes in wavenumbers $k$, the DSCRSK method is more accurate than the FD method over all the stencils examined, and is up to six orders more accurate than the FD method and the Sech method at some large stencils, see Fig. 3. For problems with considerable amplitudes in a wide range of wavenumbers as shown in Fig. 10 (b), the DSC-RSK method outperforms the FD method up to a factor of $10^{10}$, see Table 2. In differentiating $e^{i k x}$ with a relatively large wavenmuber, errors in Boyd's spectrally weighted difference, the Sech method, are up to $10^{8}$ times larger than those of the DSC-RSK method, see Fig. 1 (b). In a variety of other counterexamples, the DSC-RSK method outperforms the FD and Sech methods up to factors of multiple orders of magnitude for these problems.

Apart from the Sech and FD methods, "Proof" places great emphasis about a few other generalized finite differences, namely, Boyd's finite difference (Boyd's FD) [5], Boyd's Euler-accelerated sinc algorithm (Euler) [4, 5], and Boyd's modified Euler-accelerated sinc algorithm (MEuler) [4, 5]. Some detailed expressions and/or numerical procedures have been given in "Proof" for these methods. Moreover, some of "Proof"'s claims involve these methods. Therefore, we will perform detailed comparisons of these methods with the DSC-RSK method as well. These comparisons will enhance our understanding of high order methods, as well as the validity of "Proof"'s claims.

This paper will focus on the DSC algorithm and directly dismiss "Proof"'s claim about LDAF. In its title and abstract, "Proof" refers o LDAF as "Lagrange-distributed approximating function", while in its introduction, "Proof" refers to LDAF as "linear distributed approximating functional". The term "linear distributed approximating functional" was attributed to Hoffman et al. [13]. Unfortunately, "linear distributed approximating functional (LDAF)" simply does not exist [13]. The paper by Hoffman et al. [13] concerned the so called "distributed approximating functional" (DAF). Apart from this confusion, no single expression, no detailed analysis, nor even any correct literature reference was given to LDAF throughout "Proof". Thus, we have no clear idea what "Proof" has really proved regarding LDAF. For these reasons, we have to ignore "Proof"'s claim 
about LDAF in the rest of this paper.

The organization of this paper is the follows. In Section 2] we analyze "Proof"'s major claims in detail, while the analysis of "Proof"'s other claims will be given in Section 5 . Relevant methods and algorithms are briefly described in Section 3 Section 4 is devoted to numerical results and discussions. Finally some concluding remarks are given.

\section{Analysis of "Proof"'s central claims}

For convenience, we quote the abstract of "Proof", which contains all major claims.

- ["Proof"- AbStract] "Finite differences approximate the $m$ th derivative of a function u(x) by a series $\sum_{j=-M}^{M} d_{j}^{(m)} u\left(x_{j}\right)$, where the $x_{j}$ are the grid points. The closely-related discrete singular convolution (DSC) and Lagrange-distributed approximating function (LDAF) methods, treated here as a single algorithm, approximate derivatives in the same way as finite differences but with different numerical weights that depend upon a free parameter $a$. By means of Fourier analysis and error theorems, we show that the DSC is worse than the standard finite differences in differentiating $\exp ^{(i k x)}$ for all $k$ when $a \geq a_{\mathrm{FD}}$ where $a_{\mathrm{FD}}=1 / \sqrt{M+1}$ with $M$ as the stencil width is the value of the DSC parameter that makes its weights most closely resemble those of finite differences. For $a<a_{\mathrm{FD}}$, the DSC errors are less than finite differences for $k$ near the aliasing limit, but much, much worse for smaller $k$. Except for the very unusual case of low-pass filtered functions, that is, functions with negligible amplitude in small wavenumbers $k$, the DSC/LDAF is less accurate than finite differences for all stencil widths $M$. So-called "spectrally-weighted" or "frequency-optimized" differences are superior for this special case. Consequently, DSC/LDAF methods are never the best way to approximate derivatives on a stencil of a given width."

[AnALYSIS] First, we note that Fourier analysis is the sole technique used in "Proof". It is improper to draw conclusions regarding the performance of a numerical method for solving partial differential equations solely from its performance in differentiating $e^{i k x}$. We will elaborate on these points in Section [5.

Second, "Proof" claims that "we show that the DSC is worse than the standard finite differences in differentiating $\exp ^{(i k x)}$ for all $k$ when $a \geq a_{\mathrm{FD}}$ where $a_{\mathrm{FD}}=1 / \sqrt{M+1}$ with $M$ as the stencil width is the value of the DSC parameter that makes its weights most closely 
resemble those of finite differences". There is clearly no need to refute such a claim. Because $a$ is a free parameter, no practitioner would impose the condition $a \geq a_{\mathrm{FD}}$, but instead, an optimal choice for this parameter is sought and typically this is a value $a<a_{\mathrm{FD}}$

Third, although here "Proof" avoids specifying what is meant by "small $k$ " and what is the remainder, it does give two intervals $|K|<\frac{\pi}{2}$ and $|K|>\frac{\pi}{2}$ in Section 6 , where $K=k h$, and $h$ is the grid spacing. In Fig. 1(a), counterexamples are given to show that the DSC method outperforms the FD method at a small wavenumber, $K=\frac{9}{20} \pi$, over a wide range of stencils. Fourth, "Proof" claims that "For $a<a_{\mathrm{FD}}$, the DSC errors are less than finite differences for $k$ near the aliasing limit, but much, much worse for smaller $k$. Except for the very unusual case of low-pass filtered functions, that is, functions with negligible amplitude in small wavenumbers $k$, the DSC/LDAF is less accurate than finite differences for all stencil widths $M$ ". This claim offers great details about the use of parameter $a$, the behavior with regarding to wavenumbers $k$, the prescription of amplitude, and the behavior with respect to stencil widths. One might expect to find some verification of these statements in "Proof". Unfortunately, no verification is given. Indeed none is possible for for such a general statement. We have designed problems involving a wide range of wavenumbers, in particular, including cases whose amplitudes in small wavenumbers $k$ are either exponentially larger, or at least as large as those in large wavenumbers $k$, see Figs. 2(b), 8 (b), 10 (b) and 12 (a). Our results for these four problems are given in Fig. 3, Fig. 9, Table 2, and Fig. 12 (b), respectively. The DSC-RSK method outperforms the FD method up to $10^{6}, 10^{5}, 10^{10}$ and $10^{6}$ times, respectively for these four problems. In all four examples, the DSC outperforms the FD method over all stencil widths examined. We note that "Proof" restricted its analysis to only differentiating functions, while our last three counterexamples concern the solution of boundary value, eigenvalue and unsteady hyperbolic problems.

Fifth, for large wavenumbers, while admitting the superiority of the DSC-RSK method over the standard FD scheme, "Proof" insists that spectrally weighted differences are superior to the DSC-RSK scheme. To counter this, we just need to analyze a special case - the Sech scheme. In Fig. 1 (b), we show that for differentiating $e^{i k x}$ at $K=\frac{3}{5} \pi$, which is a function with no amplitude in small wavenumbers $k$, the DSC-RSK method is up to $10^{8}$ times more accurate than Boyd's Sech method. Another example that involves solely large wavenumbers is given in Fig. 6] Errors in the Sech scheme are up to a factor of $10^{5}$ times larger than those 
of the DSC-RSK method, see Fig. 7. In fact, in this case, the DSC-RSK method outperforms the Sech scheme over all stencil widths examined.

In fact, a large number of counterexamples exist. In Section 4 we show that up to a factor of $10^{6}$ (see Tables 1 and 2, Figs. 11](b) and 12 (b)), the DSC-RSK method outperforms the FD method and Boyd's four methods in various problems examined.

\section{Methods and algorithms}

To avoid any confusion, this section presents a brief description of the numerical methods that are studied in this work. These methods were chosen as the subject of detailed discussions in "Proof" [6], and can be expressed in the following form

$$
\frac{d^{n}}{d x^{n}} u(x) \approx \sum_{j=-M}^{M} \delta_{j}^{(n)} u\left(x+x_{j}\right)
$$

where $(2 M+1)$ is the stencil width, $x_{j}=j h$ with $h$ being the spacing, and $\delta_{j}^{(n)}$ is the differencing kernel for $n$th order derivative approximation. Note that Eq. (II) gives an interpolation approximation when $n=0$, while in general, the differentiation point $x$ may not be on-grid, i.e., $x \neq x_{j}$ for $j=-M, \ldots, M$.

Sum-accelerated pseudospectral methods are based on the Sinc kernel

$$
d_{j}^{(1), \text { sinc }}=\left\{\begin{array}{ll}
(-1)^{j+1} / h j, & j=1,2, \ldots \\
0, & j=0,
\end{array} \quad d_{j}^{(2), \text { sinc }}= \begin{cases}2(-1)^{j} / h^{2} j^{2}, & j=1,2, \ldots \\
-\pi^{2} / 3 h^{2}, & j=0 .\end{cases}\right.
$$

Various accelerations have been proposed by Boyd for on-grid (i.e., differentiation only at $x=0$ ) derivative approximation [4, 5]

$$
\frac{d^{n}}{d x^{n}} u(0) \approx \sum_{j=-M}^{M} w_{M j} d_{j}^{(\mathrm{n}), \operatorname{sinc}} u\left(x_{j}\right), \quad n=1,2,
$$

where $w_{M j}, j=-M, \ldots, M$, are the acceleration weights.

Notation of various higher order approaches is given as the follows.

- FD: the standard finite difference method. The FD weights are calculated via Fornberg's code [12].

- Boyd's FD: the finite difference method generated by applying sum acceleration, with a certain set of acceleration weights, to the standard sinc pseudospectral method [ $[5]$. The acceleration 
weights are given as

$$
w_{M j}= \begin{cases}\frac{(M !)^{2}}{(M-j) !(M+j) !}, & j=1,2, \ldots, M, \\ \frac{6}{\pi} \sum_{k=1}^{M} \frac{1}{k^{2}}, & j=0 .\end{cases}
$$

The factorial involved in sum-accelerated differences is calculated by calling the "dgamma" function of the SPECFUN package [8].

- Euler: the Euler-accelerated sinc algorithm 4, 5. The acceleration weights are given as

$$
\begin{aligned}
w_{M j} & =\sum_{k=j}^{M} \mu_{M k} \\
\mu_{M k} & =\frac{M !}{2^{n} k !(M-k) !}, \quad k=0,1, \ldots, M .
\end{aligned}
$$

- MEuler: the modified Euler-accelerated sinc algorithm [4, 5]. The acceleration weights are the same as those of Euler scheme, except that $w_{M 0}$ is modified so as to balance the contribution of all the other weights in the second order derivative approximation.

$$
w_{M 0}^{(\bmod )}=\frac{12}{\pi^{2}} \sum_{j=1}^{M} \frac{(-1)^{j+1} w_{M j}}{j^{2}} .
$$

- Sech: the spectrally weighted least squares differences with the sech weights [5]. A difference scheme is said to be "spectrally-weighted" if the kernel $\delta_{j}^{(n)}$ of Eq. (11) is chosen to minimize

$$
\int_{-\pi}^{\pi} \omega(K)\left|K^{n}-\sum_{j=-M}^{M} \delta_{j}^{(n)} \exp (i j K)\right|^{2} d K,
$$

where $K$ is the scaled wavenumber, $K=k h$. To calculate the weights, one first lets

$$
\phi_{j}(K)= \begin{cases}\sin (j K), & n \text { odd } \\ \cos (j K), & n \text { even }\end{cases}
$$

Define the matrix and vector elements

$$
G_{i j}=\int_{-\pi}^{\pi} \phi_{i}(K) \phi_{j}(K) \omega(K) d K, \quad \chi_{i}=\int_{-\pi}^{\pi} \phi_{i}(K) K^{n} \omega(K) d K .
$$

In the present study, these integrals are calculated up to double precision limit by using Gaussian quadrature with 512 nodes. The approximation accuracy is confirmed by benchmarking with the extended Simpson's rule with a very large number of nodes. The differentiation kernel is finally obtained by solving the linear system

$$
\overrightarrow{\vec{G}} \vec{\delta}=\vec{\chi}
$$


Following Boyd's suggestion [5], this least squares algebraic system is solved by using the standard singular value decomposition (SVD) solver [18].

If the Fourier spectrum of the function under study is known, one can simply choose the least squares weight function $\omega(K)$ as the Fourier spectrum, to attain an ideal approximation. However, such an information is usually unavailable in advance. Thus, the best one can do is making use of some empirical spectrum information to optimize $\omega(K)$. For example, for a smooth function whose spectrum decays exponentially with $K$ for sufficiently large $|K|$, Boyd introduced the sech weights [5]

$$
\omega(K)=\operatorname{sech}(K \pi / 2 D)
$$

where $D$ is an adjustable constant.

- Sinc: the truncated Sinc pseudospectral method, i.e., the difference kernel $\delta_{j}^{(n)}$ of Eq. (10) is chosen as $d_{j}^{(\mathrm{n}) \text {, sinc }}$ given in Eq. (2).

- DSC-RSK: the regularized Shannon kernel (RSK) of the discrete singular convolution (DSC) algorithm 22]. Although many other DSC kernels can be similarly employed, regularized Shannon kernel (RSK) 22] is employed in the present study,

$$
\delta\left(x-x_{k}\right)=\frac{\sin \frac{\pi}{h}\left(x-x_{k}\right)}{\frac{\pi}{h}\left(x-x_{k}\right)} e^{-\frac{\left(x-x_{k}\right)^{2}}{2 \sigma^{2}}}, \quad k=-M, \ldots, M,
$$

where the parameter $\sigma$ determines the width of the Gaussian envelop and often varies in association with the grid spacing, i.e., $\sigma=r h$, where $r$ is a parameter depends on the stencil parameter $M$. For given $M$, there is wide range of near optimal $r$ values to be selected. In the DSC algorithm, for derivative approximation, one first analytically differentiates the kernel

$$
\begin{aligned}
\delta^{(1)}\left(x-x_{k}\right)= & \left\{\frac{\cos \frac{\pi}{h}\left(x-x_{k}\right)}{\left(x-x_{k}\right)}-\frac{\sin \frac{\pi}{h}\left(x-x_{k}\right)}{\frac{\pi}{h}\left(x-x_{k}\right)^{2}}\right. \\
& \left.-\frac{\sin \frac{\pi}{h}\left(x-x_{k}\right)}{\frac{\pi}{h} \sigma^{2}}\right\} \exp \left(-\frac{\left(x-x_{k}\right)^{2}}{2 \sigma^{2}}\right), \\
\delta^{(2)}\left(x-x_{k}\right)= & \left\{-\frac{\frac{\pi}{h} \sin \frac{\pi}{h}\left(x-x_{k}\right)}{\left(x-x_{k}\right)}-2 \frac{\cos \frac{\pi}{h}\left(x-x_{k}\right)}{\left(x-x_{k}\right)^{2}}-2 \frac{\cos \frac{\pi}{h}\left(x-x_{k}\right)}{\sigma^{2}}\right. \\
& +2 \frac{\sin \frac{\pi}{h}\left(x-x_{k}\right)}{\frac{\pi}{h}\left(x-x_{k}\right)^{3}}+\frac{\sin \frac{\pi}{h}\left(x-x_{k}\right)}{\frac{\pi}{h}\left(x-x_{k}\right) \sigma^{2}} \\
& \left.+\frac{\sin \frac{\pi}{h}\left(x-x_{k}\right)}{\frac{\pi}{h} \sigma^{4}}\left(x-x_{k}\right)\right\} \exp \left(-\frac{\left(x-x_{k}\right)^{2}}{2 \sigma^{2}}\right) .
\end{aligned}
$$


If $x=x_{k}$, one takes the limit

$$
\delta^{(1)}(0)=0, \quad \text { and } \quad \delta^{(2)}(0)=-\frac{1}{3} \frac{3+\frac{\pi^{2}}{h^{2}} \sigma^{2}}{\sigma^{2}} .
$$

Then we generate the difference coefficients of Eq. (11) by evaluating at the point of differentiation $\delta_{j}^{(n)}=\left.\delta^{(n)}\left(x-x_{j}\right)\right|_{x}$. It is noted that only when one differentiates at $x=0$, the DSC first derivative coefficients may be rewritten in terms of the sum-acceleration difference by taking

$$
w_{M k}=\exp \left(-a^{2} k^{2}\right)
$$

where $a^{2}=\frac{1}{2 r^{2}}$. However, the second order and higher order DSC-RSK method derivative coefficients cannot be cast into such a form.

\section{Results and discussions}

To examine "Proof"'s claims in detail, we first present a comparison of the FD, Boyd's FD, Euler, MEuler, Sech and DSC-RSK methods for differentiating $e^{(i k x)}$ in Section 4.1. It is noted that $e^{(i k x)}$ involves only a single wavenumber, and might not be practical in applications. However, the subject was chosen by "Proof". In fact, most problems studied in this work involve a wide range of wavenumbers. As discussed earlier, the performance of a numerical method in differentiating $e^{(i k x)}$ does not always directly translate into its performance in differentiating other functions, and has less to do with its performance in solving a differential equation. Since the ultimate test of numerical methods is their behavior in solving differential equations, it is therefore important to examine the above mentioned methods for boundary value, initial value, eigenvalue and nonlinear problems. It is noted that a boundary value problem studied here was in fact previously designed by Boyd for comparing the performances of the FD, Euler, and Sech methods [ $[$.

Both the Sech and DSC-RSK methods have an adjustable parameter. For the Sech method, the parameter $D$ depends on the spectral property of the solution. Thus, for a fixed problem, it is independent of the half band-width $M$, while the parameter $r$ of the RSK depends on $M$. In the following studies, the numerically-tested optimal $r$ and $D$ will be chosen, and will be reported. In boundary value problems, exact values outside the computational domain are used to impose boundary conditions. 
(a)

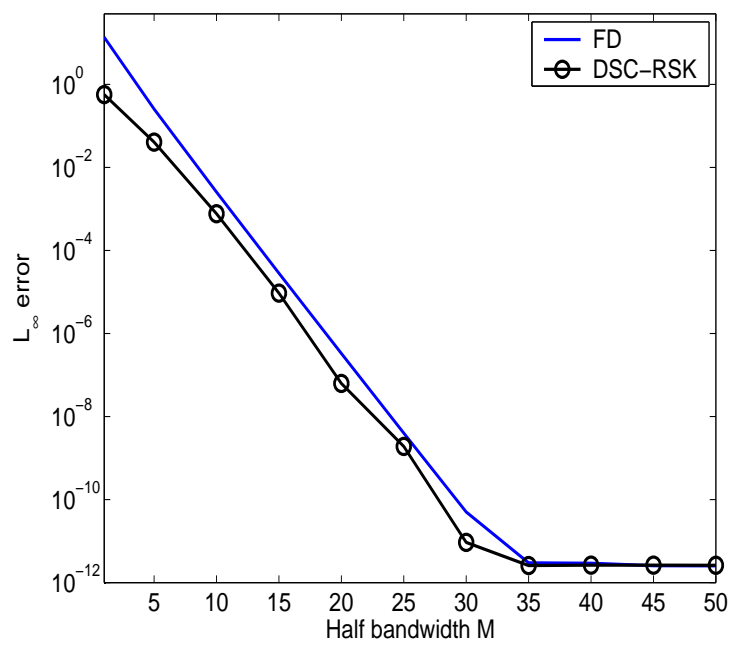

(b)

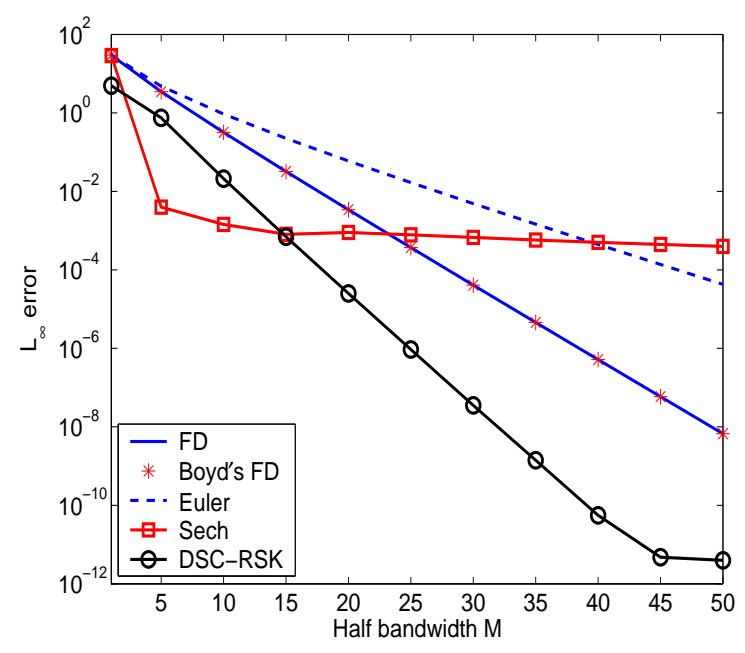

Figure 1: Error analysis in differentiating $\exp (i k x)$. (a) $k h=\frac{9}{20} \pi$; (b) $k h=\frac{3}{5} \pi$.

\subsection{Differentiating $e^{i k x}$}

We first consider the approximation of differentiating $u(x)=\exp (i k x)$. It is claimed in "Proof" that the DSC is more accurate than the FD only when $k h>\frac{\pi}{2}$. A counterexample can be easily constructed at $k h=\frac{9}{20} \pi$ by considering a grid of 201 evenly spaced points in the interval of $x \in[0,2 \pi]$ and setting $k=45$. A near optimal DSC-RSK parameter $r$ is used in our computation for each $M:(M, r)=(1,1.2),(5,1.9),(10,2.7),(15,3.2),(20,3.6),(25,4.0),(30,4.3),(35,4.7),(40,5.1)$, $(45,5.5)$, and $(50,5.9) . L_{\infty}$ errors are depicted in Fig. 1 (a). The numerical results clearly indicate that the DSC-RSK errors are smaller than those of the FD method for a wavenumber less than $\frac{\pi}{2 h}$, albeit the FD method is usually more accurate when $k$ is much smaller. This gives rise to our first counterexample of "Proof".

Ironically, these results were partially implied in Fig. 2 of "Proof", plotted with $M=33$. However, the results presented here indicate that the DSC-RSK method outperforms the finite difference for many small $M$ values, ranging from 1 to 35 .

We next consider a problem with a medium-high wavenumber, $k h=\frac{3}{5} \pi$, by increasing $k$ to 60 in the last case. This wavenumber is still far away from the aliasing limit. A near optimal Sech parameter, $D=0.17$ is used for all $M$ from 1 to 50 , while $r$ for the DSC-RSK method is chosen as: $(M, r)=(1,2.3),(5,2.4),(10,3.1),(15,3.7),(20,4.2),(25,4.7),(30,5.1),(35,5.5),(40,5.8),(45,6.2)$, and $(50,6.5) . L_{\infty}$ errors are depicted in Fig. 1 (b). The errors of the FD and Boyd's FD methods are identical. The Sech method has a better performance only for $1<M<15$. Its accuracy 
(a)

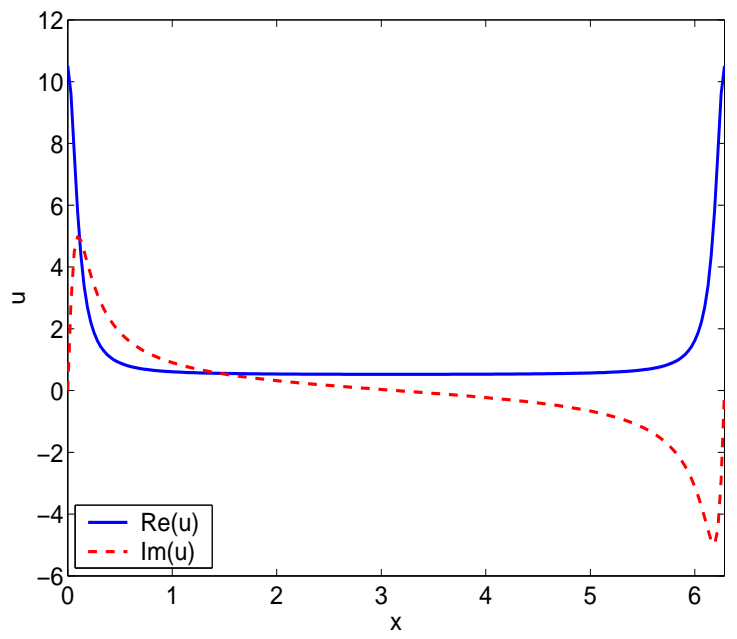

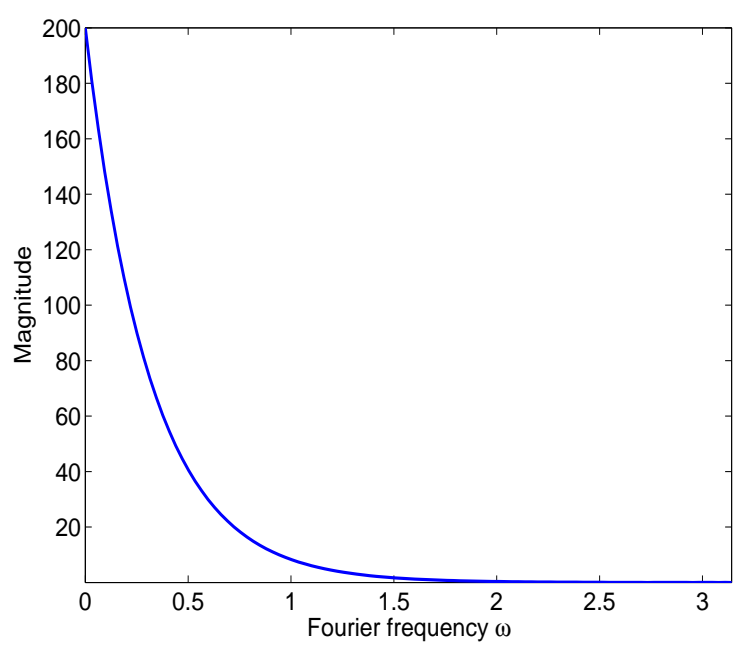

Figure 2: (a) The function with exponentially decaying amplitudes in wavenumbers; (b) Its Fourier frequency response.

does not improve as $M$ is increased beyond $M=15$. Therefore, if $L_{\infty}$ error is required to be less than $10^{-4}$ for this problem, the Sech method cannot make it, whereas all other methods have a chance. The DSC-RSK method clearly outperforms the FD method up to 1000 times, and Boyd's spectrally-weighted finite difference (Sech) method up to $10^{8}$ times at some large stencils. This gives to another counterexample to "Proof".

\subsection{Differentiating a function having exponentially decaying amplitudes in wavenum- bers $k$}

Although high frequency problems are common in science and engineering, some physical problems have their spectral distribution centered in the low frequency part, i.e., their amplitudes in large wavenumbers decay exponentially. Therefore, we consider the approximation of differentiating such a function, which can be constructed as a weighted summation of Fourier basis functions $\exp (i k x)$

$$
u(x)=\sum_{k=0}^{k_{\max }} \exp (-k \sigma) \exp (i k x), \quad x \in[0,2 \pi] .
$$

Here, we choose $\sigma=0.1$ and $k_{\max }=80$. We carry out the differentiation on a grid of 201 evenly spaced points. A near optimal Sech parameter, $D=0.17$ is used for all $M$ from 1 to 80, while $r$ for the DSC-RSK method is chosen as: $(M, r)=(1,0.9),(5,1.8),(10,2.9),(15,3.8),(20,4.6),(25,5.3)$, $(30,5.9),(35,6.5),(40,7.1),(45,7.6),(50,8.1),(55,8.1),(60,9.0),(65,9.4),(70,9.8),(75,10.3)$, and 


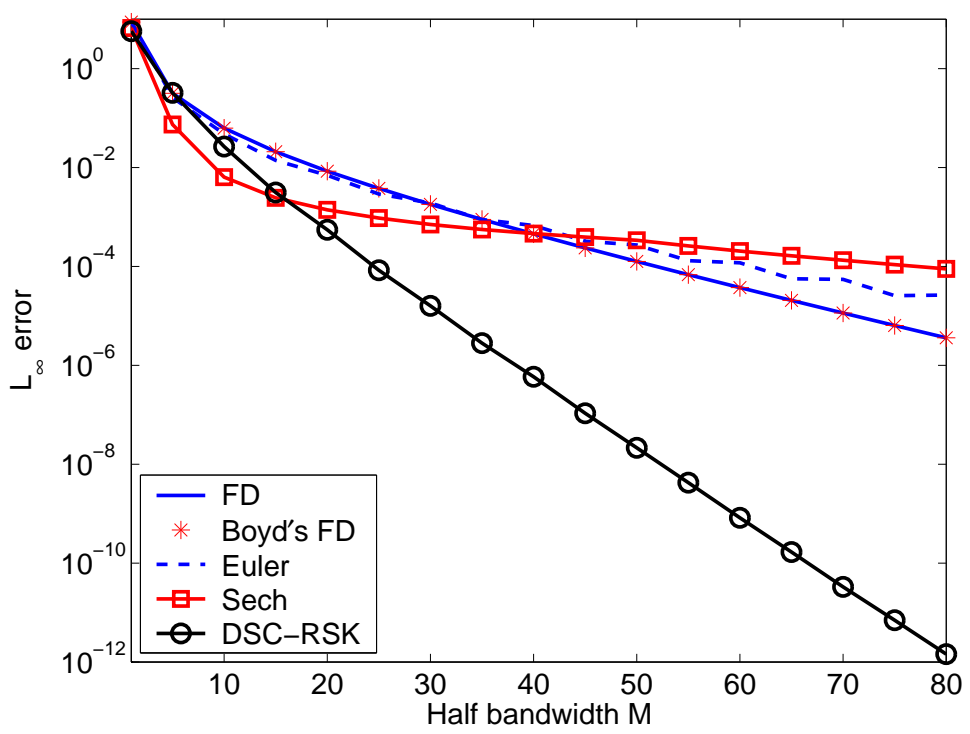

Figure 3: Error analysis in differentiating the function having exponentially decaying amplitudes in wavenumbers.

$(80,10.6)$.

"Proof" claims that "Except for the very unusual case of low-pass filtered functions, that is, functions with negligible amplitude in small wavenumbers $k$, the DSC/LDAF is less accurate than the FD for all stencil widths $M$ ". In the present problem, amplitudes in wavenumbers decay exponentially. Fig. 2 gives the function and its Fourier frequency response. Fig. 3] depicts the error analysis of various numerical methods. Contrary to "Proof"'s claim, the DSC-RSK method is more accurate than finite differences for all stencil widths $M$ considered. The Sech method performs better only when $1<M<15$, and becomes the worst for large $M$ values. The DSC-RSK method clearly outperforms the FD method up to $10^{6}$ times, and the Sech method up to $10^{8}$ times at some large stencils. 


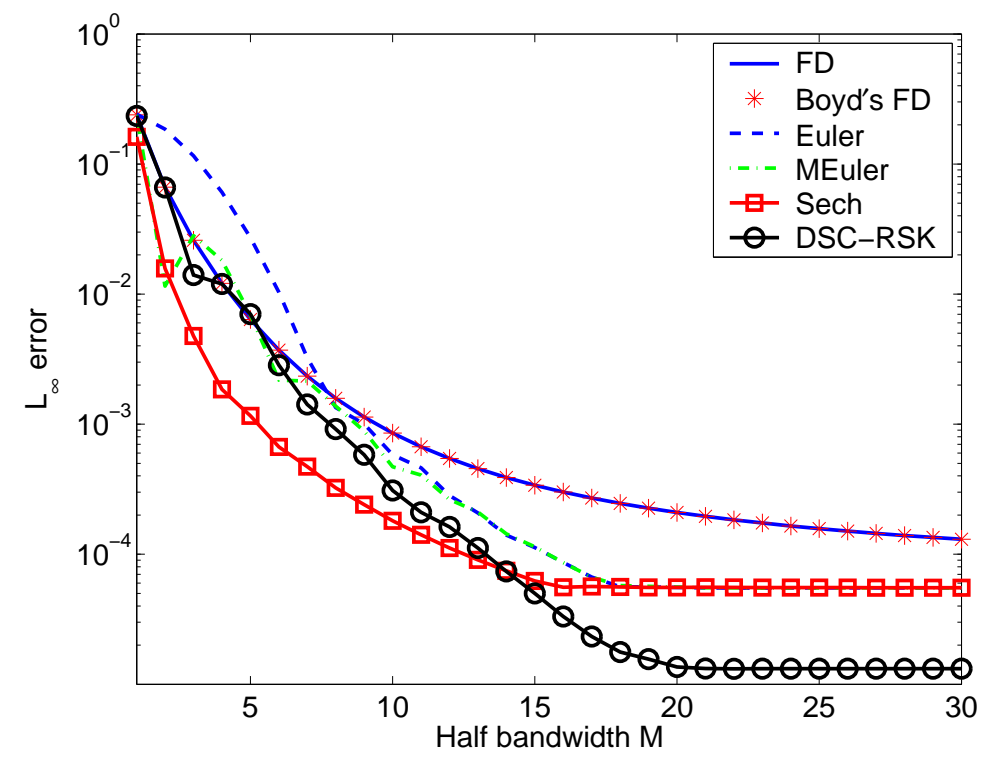

Figure 4: Error analysis of Boyd's boundary value problem.

\subsection{Boyd's boundary value problem}

Boyd employed a boundary value problem [5] to demonstrate the superior performance of his spectrally-weighted difference, the Sech method, over the FD scheme

$$
\begin{aligned}
u_{x x}-u & =f \\
f & =\left\{-2 \operatorname{sech}^{3}(x)-\frac{\pi^{2}}{4 h^{2}} \operatorname{sech}(x)\right\} \cos \left(\frac{\pi x}{2 h}\right)+\frac{\pi}{h} \operatorname{sech}(x) \tanh (x) \sin \left(\frac{\pi x}{2 h}\right), \\
u & =\operatorname{sech}(x) \cos \left(\frac{\pi x}{2 h}\right),
\end{aligned}
$$

where $h$ is the spacing. A grid of 201 evenly spaced points is used spanning the interval $x \in[-30,30]$, which gives $h=0.3$. The analytical solution outside the computational domain was employed to impose boundary conditions. Boyd examined the behavior of numerical methods by varying the stencil width $2 M+1$ from $M=1$ to 20 , while the results for the Sech method was only given from $M=1$ to 8 . A near optimal Sech parameter, $D=0.25$ was used for all $M$ (see [5]). In this work, we shall solve the problem also extending the upper limit of the stencil parameter from $M=20$ to 30. A near optimal DSC-RSK parameter $r$ is used in our computations for each $M$ : $(M, r)=(1,2.3),(2,1.4),(3,1.7),(4,2.2),(5,2.4),(6,2.5),(7,2.6),(8,2.9),(9,3.1),(10,3.3),(11,3.5)$, $(12,3.7),(13,4.0),(14,4.1),(15,4.3),(16,4.5),(17,4.7),(18,4.9),(19,5.1),(20,5.4),(21,5.4),(22,5.4)$, $(23,5.4),(24,5.4),(25,5.4),(26,5.4),(27,5.4),(28,5.4),(29,5.4)$, and $(30,5.4)$. 
(a)

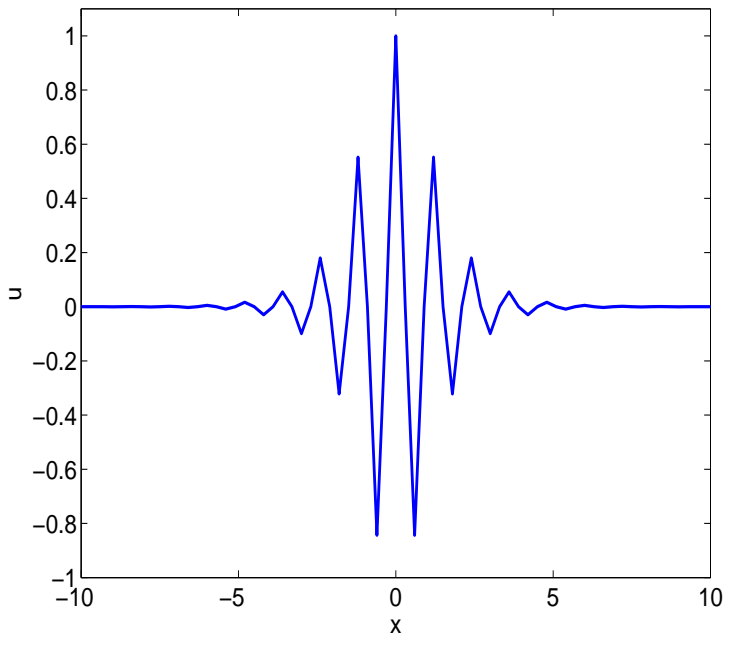

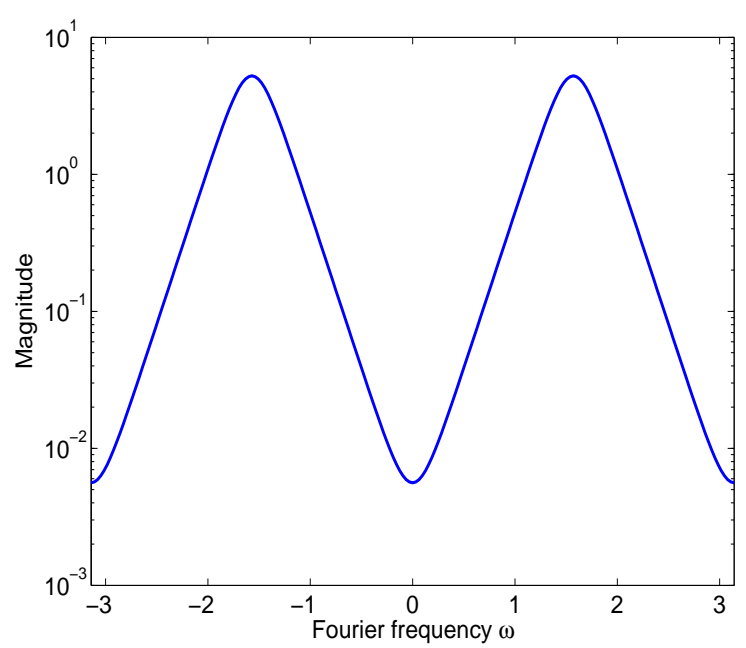

Figure 5: (a) Solution of Boyd's boundary value problem; (b) Its Fourier frequency response.

$L_{\infty}$ errors are depicted in Fig. 4. We note that the behaviors of FD, Euler, and Sech methods are identical to those in Fig. 4 of Ref. [5]. Indeed, for $M<13$ the Sech method performs better than the FD and DSC-RSK schemes. However, for a large stencil width, the DSC-RSK method clearly outperforms all other methods, including the FD and Sech methods. Therefore, Boyd's boundary value problem is a counterexample of his claims in "Proof".

The flat and comparatively large errors of all six different methods for $M \geq 20$ are suspicious, indicating that the errors are limited by the original design of the problem. To investigate further, we analyzed the Fourier frequency response of the exact solution as shown in Fig. [5 (b). Indeed, the magnitude of the Fourier frequency response has a large truncation at $|\pi|$, which gives rise to inherent aliasing errors. Therefore, this problem is not appropriate for demonstrating the full potential of different numerical methods.

\subsection{Boundary value problem with a confined distribution of wavenumbers}

Since the above boundary value problem, taken from [5], cannot reveal the true accuracy of numerical methods, let us consider a modification

$$
\begin{aligned}
u_{x x}-u & =f, \quad x \in[-3,3], \\
u & =\exp \left(-\frac{x^{2}}{2 a^{2}}\right) \cos (b \pi x), \\
f & =\frac{2 b \pi x}{a^{2}} \exp \left(-\frac{x^{2}}{2 a^{2}}\right) \sin (b \pi x)+\left(\frac{x^{2}}{a^{4}}-\frac{1}{a^{2}}-(b \pi)^{2}-1\right) \exp \left(-\frac{x^{2}}{2 a^{2}}\right) \cos (b \pi x) .
\end{aligned}
$$


(a)

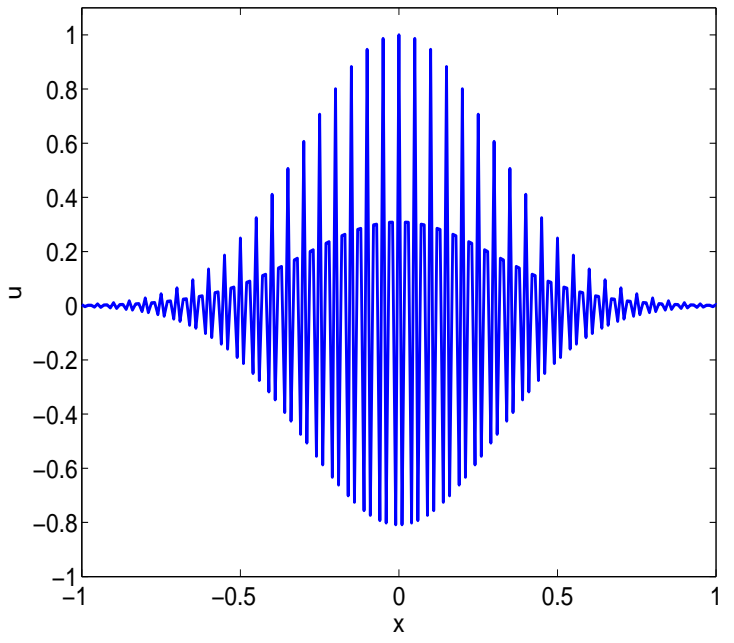

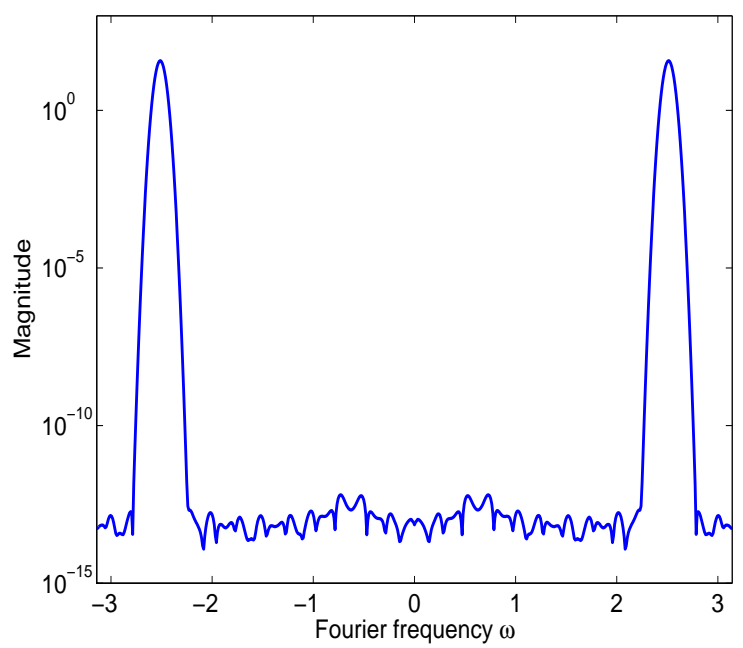

Figure 6: (a) The solution of a boundary value problem with a confined distribution of wavenumbers; (b) Its Fourier frequency response.

By choosing $a=0.3$ and $b=80$, the solution is oscillatory and its frequency response shows a peak around \pm 2.5 , see Fig. 6. Note that the frequency magnitude is as small as $10^{-14}$ near $\pm \pi$. Therefore, in contrast to the previous boundary value problem, the present problem has negligible inherent aliasing error. Due to the frequency distribution in the large wavenumber region, this case favors numerical methods that perform well for large wavenumbers. We employ a grid of $N=601$ points in the interval $x \in[-3,3]$. In the Sech method, the nearly optimized $D=0.18$ is used. Other $D$ could be slightly better but the amount of improvement would be not significant enough to alter the conclusion of our analysis. For the DSC-RSK method, near optimal $r$ values are chosen as $(M, r)=(1,2.7),(5,3.9),(10,4.5),(15,6.0),(20,6.4),(25,7.1),(30,7.6),(35,8.1),(40,8.6),(45,9.2)$, $(50,9.6),(55,10.1),(60,10.5),(65,11.0),(70,11.3),(75,11.8)$, and $(80,12.3)$.

Since this is a problem involving large wavenumbers, according to "Proof", the spectrallyweighted differences are designed for handling this kind of problems, and will outperform the DSC-RSK method. Fig. 7 illustrates the $L_{\infty}$ errors for six different numerical methods for this case. It is interesting to note that Boyd's FD method yields the same results as the standard FD method for all $M$ considered. The MEuler method is slightly more accurate than the Euler method when $M$ is relatively small, while when $M>10$, their results are identical. The Sech method is more accurate than these four differencing methods over a wide range of $M$ for this high frequency problem. However, it is clear from Fig. 7 that the DSC-RSK method is the most 


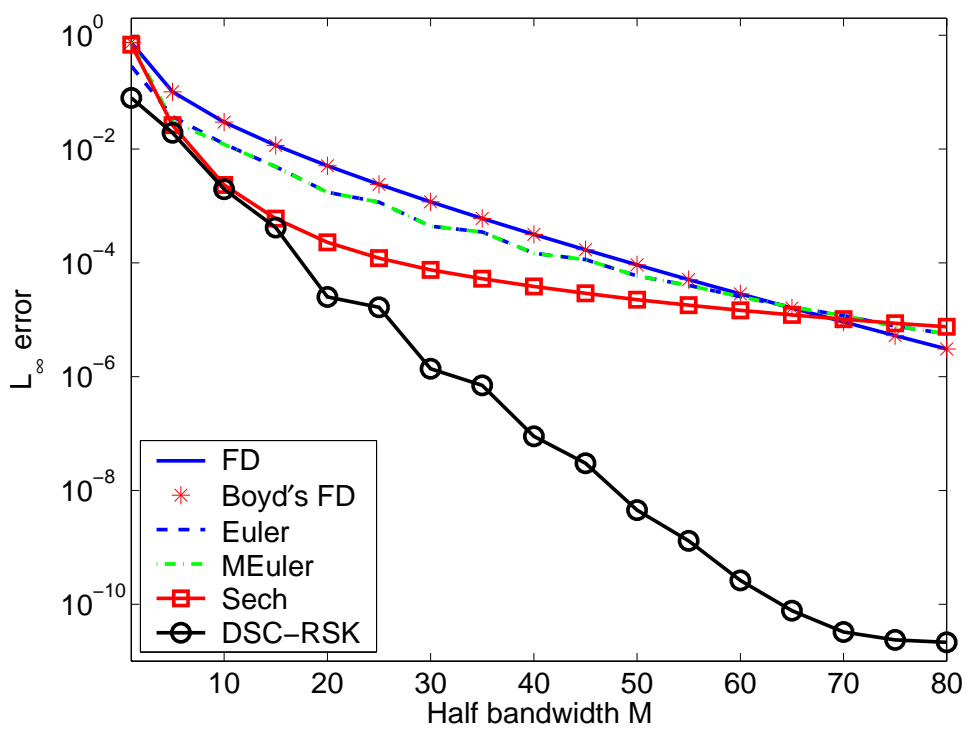

Figure 7: Error analysis for the boundary value problem with confined wavenumber distribution.

accurate method for all $M$ values. In particular, when $M=80$, the DSC-RSK method is about five orders more accurate than any other numerical method considered. In fact, at large $M$, the accuracy of the DSC-RSK method, $L_{\infty}=10^{-11}$, is limited by the iterative algebraic solver, the standard preconditioned biconjugate gradient (PBCG) method [18].

\subsection{Boundary value problem with a wide range of wavenumbers}

It is important to study problems with a wide range of wavenumbers. Let us consider a boundary value problem

$$
\begin{aligned}
u_{x x}-u & =f, \quad x \in[-3,3] \\
u & =\sum_{b=1}^{8} \exp \left(-\frac{x^{2}}{2 a^{2}}\right) \cos (10 b \pi x) \\
f & =\sum_{b=1}^{8} \frac{20 b \pi x}{a^{2}} \exp \left(-\frac{x^{2}}{2 a^{2}}\right) \sin (10 b \pi x)+\sum_{b=1}^{8}\left(\frac{x^{2}}{a^{4}}-\frac{1}{a^{2}}-(10 b \pi)^{2}-1\right) \exp \left(-\frac{x^{2}}{2 a^{2}}\right) \cos (10 b \pi x),
\end{aligned}
$$

where $a=0.3$. The solution and its frequency response are depicted in Fig. 8. It is seen that the frequency response has a wide range of wavenumbers. We have arranged the amplitudes in small wavenumbers to be as large as those in large wavenumbers (see Figs. 8 (b)).

We still solve the problem with $N=601$. For the Sech method, it is not easy to find the optimal parameter $D$ for this problem due to a wide range of involved wavenumbers. A near optimized 
(a)

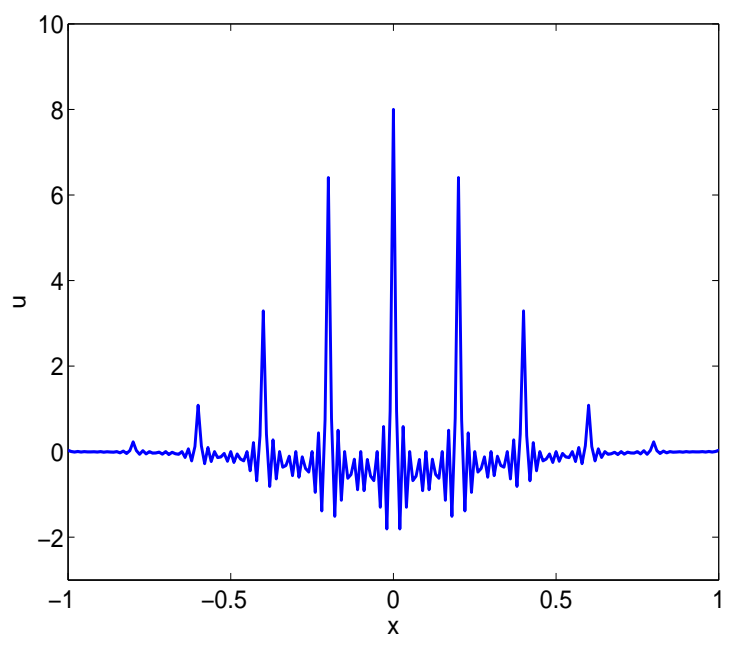

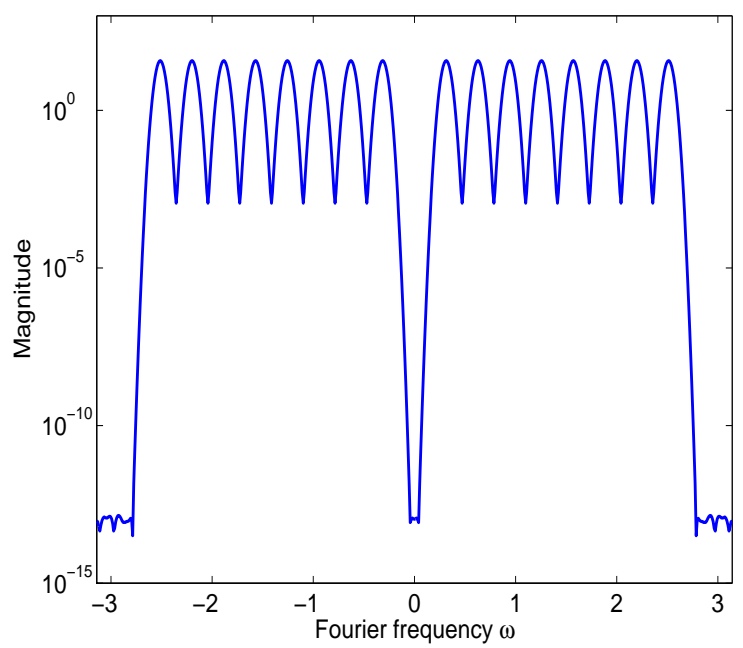

(b)

Figure 8: (a) The solution of a boundary value problem with a wide range of wavenumbers; (b) Its Fourier frequency response.

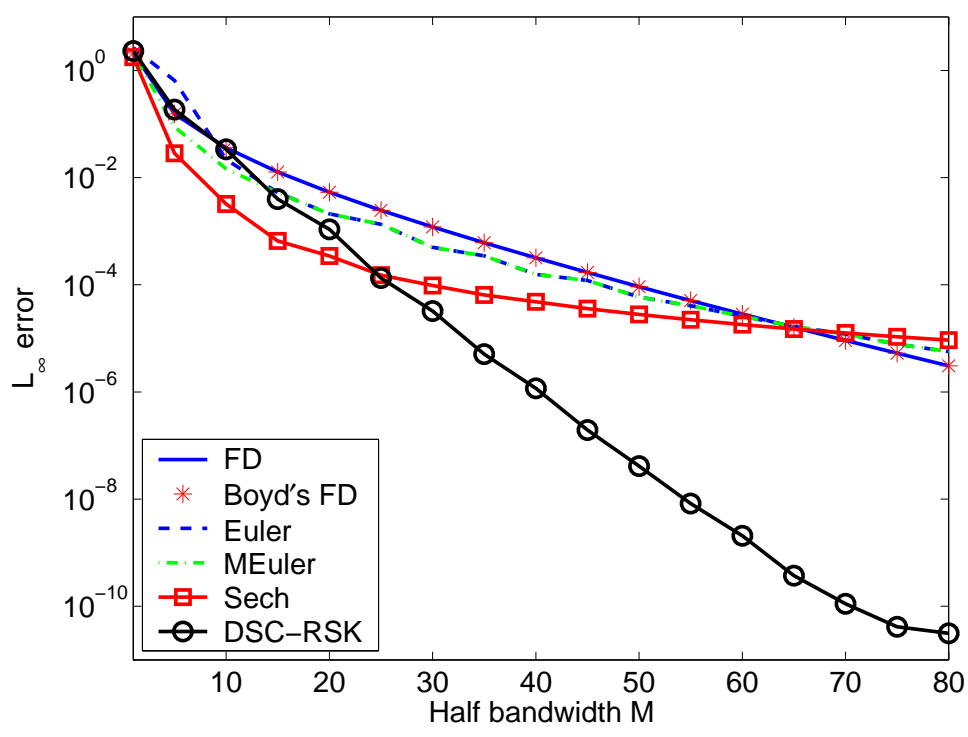

Figure 9: Error analysis for the boundary value problem with a wide range of wavenumbers

value, $D=0.18$, is employed. For the DSC-RSK method, near optimal parameters are as follows: $(M, r)=(1,1.1),(5,2.6),(10,3.1),(15,4.6),(20,5.3),(25,6.1),(30,6.8),(35,7.5),(40,8.1),(45,8.5)$, $(50,9.0),(55,9.5),(60,10.0),(65,10.4),(70,10.8),(75,11.4)$, and $(80,11.8)$.

The $L_{\infty}$ errors are given in Fig. 9 for different $M$. The MEuler method is more accurate than the Euler method for small $M$, while when $M>15$, their results are identical. For a small $M$, such 
as up to 80 in this example, Boyd's FD method yields the same results as the FD method. However, when $M>80$, Boyd's FD method is less accurate, probably because for large $M$, calculating the weights of Boyd's FD method produces numerical errors. The results of the Sech method are similar to those in the last case. In particular, the Sech method is more accurate than the FD, Boyd's FD, Euler and MEuler methods when $M<70$, while when $M$ is sufficient large, the accuracy of the Sech method is worse than the others. It is noted that the Sech method performs slightly better than the DSC-RSK method from $M<25$, due to large amount of the small wavenumber components. However, for a large $M$, the DSC-RSK method is much more accurate.

"Proof" claims that "For $a<a_{\mathrm{FD}}$, the DSC errors are less than finite differences for $k$ near the aliasing limit, but much, much worse for smaller $k$. Except for the very unusual case of lowpass filtered functions, that is, functions with negligible amplitude in small wavenumbers $k$, the DSC/LDAF is less accurate than finite differences for all stencil widths $M$ ". The present example satisfies all of "Proof"'s descriptions, except for the conclusion: the DSC-RSK method is more accurate than the FD method for all stencil widths examined. When $M=80$, the DSC-RSK method outperforms the FD scheme and all other methods by more than 100,000 times. If high accuracy is desirable for this problem, the DSC-RSK scheme is the method of choice among the six methods.

\subsection{Helmholtz equation with a constant source term}

We next consider the Helmholtz equation with high wavenumbers $k$

$$
\begin{aligned}
-u_{x x}(x)-k^{2} u(x) & =1, \quad x \in[0,1] \\
u(0) & =0 \\
u_{x}(1)-i k u(1) & =0 \\
u(x) & =\frac{1}{k^{2}}[(1-\cos (k x)-\sin (k) \sin (k x))+i(\cos (k)-1) \sin (k x)] .
\end{aligned}
$$

This problem has its origin from electromagnetics and is notoriously challenging when $k$ is large for a given number of grid points (see [1, 2, 9, 14, 15]). High wavenumbers are common in electricallylarge systems. As "Proof" claims that spectrally-weighted differences are superior to the DSC for

differentiating functions with large wavenumbers, we employ the Sech method. We shall explore the capability of each high order method for resolving high frequency problems with a small mesh density close to the Nyquist limit. A method that can achieve a spatial resolution near the Nyquist 
(a)

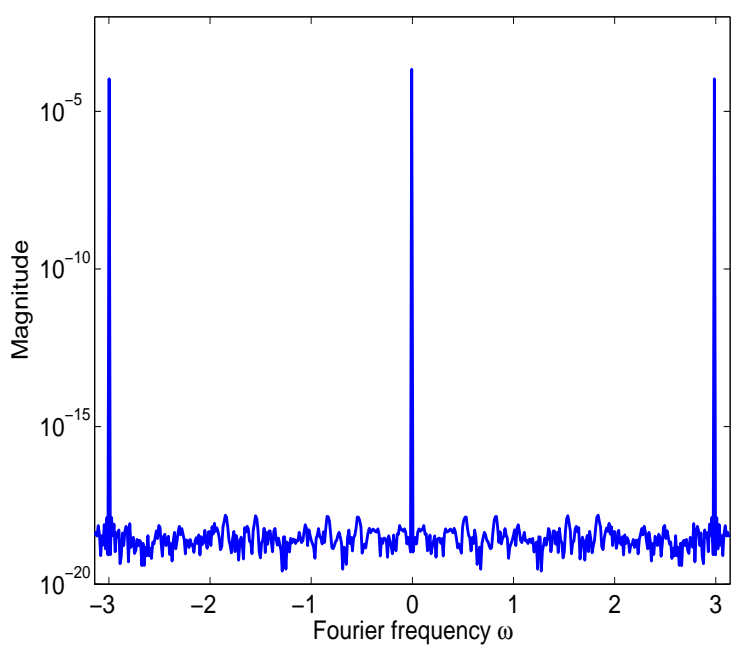

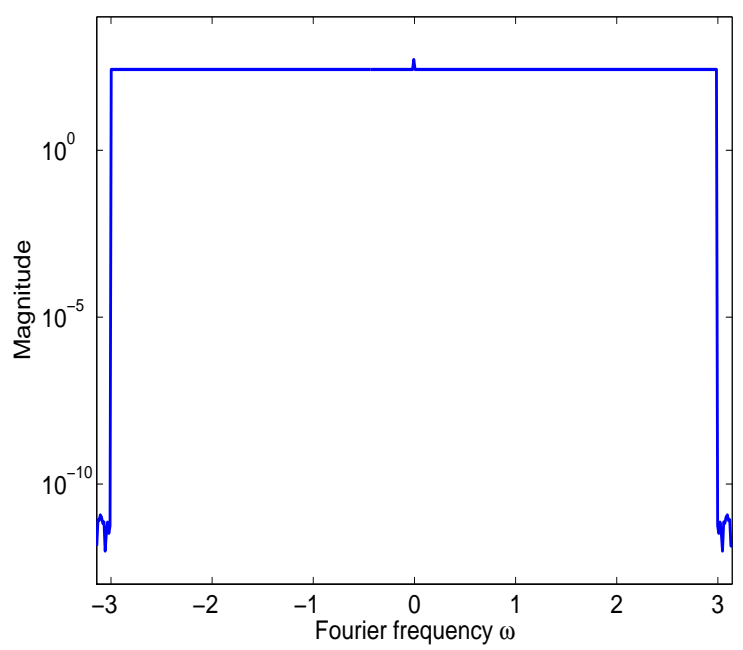

(b)

Figure 10: Fourier frequency response of the solution to the Helmholtz equation with high wavenumbers. (a) With a constant source; (b) With a multiple frequency source.

limit, 2 points per wavelength (PPW), without invoking much alias errors, is more efficient for solving large scale problems. The DSC-RSK method was applied to this problem in our previous study [3]. The objective of the present work is to examine "Proof"'s claim and to explore different numerical methods.

We first consider the case of $k=500 \pi$. The Fourier frequency response of the solution is depicted in Fig. 10. We use a grid of $N=526$ points in the domain so that the grid density is about 2.1 PPW, which is very close to the Nyquist sampling limit. Therefore, the setting of problem is extremely difficult for numerical methods that do not work well for high wavenumbers. The $H^{1}$-seminorm will be used to measure the error 3 ]

$$
e_{1}:=\frac{\left|u-u_{h}\right|_{1}}{|u|_{1}}
$$

where $u_{h}$ is the numerical approximation to the solution $u$, and $|u|_{1}=\left\|\frac{d u}{d x}\right\|$. Note that for this problem, large stencils are required to deliver reasonable results. We solve the problem with a variety of $M$ values ranging from $M=50$ to 500. Since the values of $M$ are quite large, the total execution time for generating the first and second derivative weights is also reported for each method for a comparison. In the Sech method, a near optimized parameter $D=0.28$ is used for all $M$. In the DSC-RSK method, we choose $(M, r)=(50,9.0),(100,27.9),(150,35.1),(200,38.1)$, $(250,42.3),(300,46.9),(350,49.6),(400,53.5),(450,56.0)$, and $(500,60.0)$.

Since the numerical errors vary over 160 orders, it is inconvenient to put them in a figure. We 
therefore present them in Table [. It is seen from the table that for the present problem with $\mathrm{PPW}=2.1$, the FD method does not improve much when $M$ is increased from 50 to 500 . Its errors are intolerably large for all $M$ values. Therefore, the FD method is not efficient for this highly oscillatory problem.

When $M=50$, Boyd's FD scheme yields the same results as the standard FD method. However, when $M=100$, although the weights are generated in 0.0003 second, the rest of the computation lasts a very long time without delivering any reasonable result. It gets worse for $M>100$. This seems to suggest that Boyd's FD method is unstable for large $M$.

Although the Sech method was proposed to deal with high wavenumbers, it does not work the way as promised for very high wavenumber problems. One major problem with the Sech method is that it requires excessive CPU time for generating the weights. Since the Gram matrix is poorly conditioned, the SVD solver is often used, which is CPU demanding. Thus, the Sech method seems to be impractical for high frequency problems. Moreover, the SVD solver [18 fails to converge when $M \geq 300$ for the Sech method, probably because the matrix is seriously ill-conditioned for large $M$. Except for $M=50$, the Sech method was less accurate than the DSC-RSK method for this challenging problem. At $M=250$, the DSC-RSK method is about $10^{8}$ times more accurate than the Sech method. If the minimal accuracy requirement is $10^{-3}$, no other methods except for the DSC-RSK method can be used for this problem.

The Euler and MEuler methods produce identical results. Their errors reach $10^{149}$ at $M=500$, indicating the instability in computing the factorial. Nevertheless, the same sets of weights seem to work better in the next problem with a wide range of high wavenumbers. Therefore, these two methods are not stable for being used in high wavenumber problems from the Helmholtz equation.

Out of six approaches, the DSC-RSK method is the only method that is able to deliver extremely accurate solutions at grid density of $2.1 \mathrm{PPW}$ for this problem. It also requires the smallest CPU time for the generation of the weights.

\subsection{Helmholtz equation with a multiple frequency source term}

Here we compare the FD and DSC methods when there is significant amplitude in a wide range of wavenumbers. To this end, we consider the Helmholtz equation with a multiple frequency source 
Table 1: Numerical results and CPU times in seconds for solving the Helmholtz equation with a constant source term. Here NaN and - stand for "not a number" and "not available", respectively.

\begin{tabular}{|c|c|c|c|c|c|c|}
\hline & \multicolumn{2}{|c|}{ FD } & \multicolumn{2}{|c|}{ Boyd's FD } & \multicolumn{2}{|c|}{ Sech } \\
\hline$M$ & Error & $\mathrm{CPU}$ & Error & $\mathrm{CPU}$ & Error & $\mathrm{CPU}$ \\
\hline 50 & $9.98(-1)$ & $8.63(-4)$ & $9.98(-1)$ & $4.49(-4)$ & $5.71(-2)$ & 3.983 \\
\hline 100 & $2.32(+0)$ & $3.13(-3)$ & $\mathrm{NaN}$ & $3.41(-4)$ & $5.24(-1)$ & 15.69 \\
\hline 150 & $1.17(+0)$ & $7.02(-3)$ & - & - & $8.88(-2)$ & 35.25 \\
\hline 200 & $1.36(+0)$ & $1.38(-2)$ & - & - & $3.98(-2)$ & 62.77 \\
\hline 250 & $4.27(+0)$ & $2.03(-2)$ & - & - & $4.85(-2)$ & 98.26 \\
\hline 300 & $6.57(-1)$ & $2.84(-2)$ & - & - & - & - \\
\hline 350 & $5.09(-1)$ & $3.88(-2)$ & - & - & - & - \\
\hline 400 & $4.10(-1)$ & $4.91(-2)$ & - & - & - & - \\
\hline 450 & $3.23(-1)$ & $6.17(-2)$ & - & - & - & - \\
\hline \multirow[t]{2}{*}{500} & $2.49(-1)$ & $7.58(-2)$ & - & - & - & - \\
\hline & \multicolumn{2}{|c|}{ Euler } & \multicolumn{2}{|c|}{ MEuler } & \multicolumn{2}{|c|}{ RSK } \\
\hline$M$ & Error & $\mathrm{CPU}$ & Error & $\mathrm{CPU}$ & Error & $\mathrm{CPU}$ \\
\hline 50 & $2.72(-1)$ & $4.77(-4)$ & $2.72(-1)$ & $4.72(-4)$ & $1.16(+0)$ & $3.06(-4)$ \\
\hline 100 & $2.94(+1)$ & $4.01(-4)$ & $2.94(+1)$ & $4.08(-4)$ & $1.12(-2)$ & $2.31(-4)$ \\
\hline 150 & $3.02(-1)$ & $5.95(-4)$ & $3.02(-1)$ & $6.16(-4)$ & $7.52(-5)$ & $3.39(-4)$ \\
\hline 200 & $5.16(+58)$ & $5.83(-4)$ & $5.16(+58)$ & $7.30(-4)$ & $2.27(-8)$ & $4.20(-4)$ \\
\hline 250 & $5.81(+73)$ & $6.86(-4)$ & $5.81(+73)$ & $7.02(-4)$ & $7.34(-10)$ & $5.55(-4)$ \\
\hline 300 & $6.55(+88)$ & $2.67(-2)$ & $6.55(+88)$ & $2.67(-2)$ & $7.08(-10)$ & $6.57(-4)$ \\
\hline 350 & $7.37(+103)$ & $2.04(-2)$ & $7.37(+103)$ & $2.08(-2)$ & $3.45(-11)$ & $7.15(-4)$ \\
\hline 400 & $8.30(+118)$ & $2.56(-2)$ & $8.30(+118)$ & $2.65(-2)$ & $2.83(-13)$ & $8.09(-4)$ \\
\hline 450 & $9.35(+133)$ & $2.74(-2)$ & $9.35(+133)$ & $2.82(-2)$ & $1.50(-13)$ & $9.05(-4)$ \\
\hline 500 & $1.05(+149)$ & $3.64(-2)$ & $1.05(+149)$ & $3.64(-2)$ & $1.77(-13)$ & $9.97(-4)$ \\
\hline
\end{tabular}

term

$$
\begin{aligned}
u_{x x}(x)+k^{2} u(x) & =f(x), \quad x \in[0, \pi] \\
u(0) & =u(\pi)=\frac{k}{2}+1, \\
f(x) & =\sum_{j=0}^{k / 2-1}\left(k^{2}-4 j^{2}\right) \cos (2 j x), \\
u(x) & =\sum^{k / 2} \cos (2 j x) .
\end{aligned}
$$


By considering $N=526$ and $k=500$, the grid density of this problem is also PPW=2.1. The Fourier frequency response of the solution is depicted in Fig. 10 (b). This is a problem that involves almost all available spectrum of the discrete Fourier domain. It is a good example to illustrate "Proof"'s claim.

Since this problem requires a large stencil, we examine the performance of different methods over a range of large $M$ values, from $M=50$ to 500. For the Sech method, the near optimized $D=0.28$ is used for all $M$. For the DSC-RSK method, we choose $(M, r)=(50,9.0),(100,27.9)$, $(150,35.1),(200,38.1),(250,42.3),(300,46.9),(350,49.6),(400,53.5),(450,56.0)$, and $(500,60.0)$

$L_{\infty}$ errors are listed in Table 2. Execution time for generating the first and second derivative weights is also reported for each approach. These results are similar to those in the previous example. The Euler and MEuler methods show a great deal of improvement from their last performance. However, their results are still up to 12 orders less accurate than those of the DSC-RSK method at certain $M$ values.

For this multiple frequency problem, it is noted that the accuracy of the FD method does not improve much as the $M$ is increased from 50 to 400. It fails to converge when $M>400$, indicating the possible instability in the FD method for this class of multiple frequency problems. The DSCRSK method outperform the FD method by up to 10 orders of magnitude. Out of six methods studied, the DSC-RSK method is the only one that is able to deliver high accuracy for this multiple frequency problem.

\subsection{Unsteady hyperbolic equation with a few wavenumbers}

We consider the time-dependent equation

$$
\begin{aligned}
u_{t} & =-t^{2} u_{x}, \quad x \in[-1,1] \\
u(0, x) & =\sin ^{4}(k \pi x), \\
u(t, x) & =\sin ^{4}\left(k \pi\left(x-t^{3} / 3\right)\right),
\end{aligned}
$$

with periodical boundary condition. Fourier frequency response of $u(0, x)$ is given in Fig. 11 (a).

We use the classical fourth order Runge-Kutta (RK4) scheme for the time discretization. To compare the spatial discretization errors of various higher order approaches, a sufficiently small 
Table 2: Numerical results and CPU times in second for solving the Helmholtz equation with a multiple frequency source term. Here NaN and - stand for "not a number" and "not available", respectively.

\begin{tabular}{lllllll}
\hline \hline & \multicolumn{3}{c}{ FD } & \multicolumn{2}{c}{ Boyd's FD } & \multicolumn{2}{c}{ Sech } \\
\hline$M$ & Error & CPU & Error & CPU & Error & CPU \\
\hline 50 & $5.06(+0)$ & $8.92(-4)$ & $5.06(+0)$ & $4.88(-4)$ & $1.78(-1)$ & 3.998 \\
100 & $5.48(+0)$ & $3.12(-3)$ & NaN & $3.45(-4)$ & $1.51(-1)$ & 15.73 \\
150 & $2.37(+0)$ & $7.02(-3)$ & - & - & $4.76(-2)$ & 35.38 \\
200 & $4.06(+0)$ & $1.23(-2)$ & - & - & $2.07(-2)$ & 62.98 \\
250 & $1.14(+0)$ & $1.91(-2)$ & - & - & $2.75(-2)$ & 98.56 \\
300 & $6.60(-1)$ & $2.75(-2)$ & - & - & - & - \\
350 & $4.27(-1)$ & $3.83(-2)$ & - & - & - & - \\
400 & $2.87(-1)$ & $4.89(-2)$ & - & - & - & - \\
450 & NaN & $7.20(-2)$ & - & - & - & - \\
500 & NaN & $1.78(-1)$ & - & - & - & - \\
\hline & & & & & & \\
\hline
\end{tabular}

time increment $\Delta t$ has to be used. Here, we choose $N=101, k=10, t=1$, and $\Delta t=5 \times 10^{-5}$. It was numerically tested that a smaller $\Delta t$ would not yield significantly more accurate results. In 

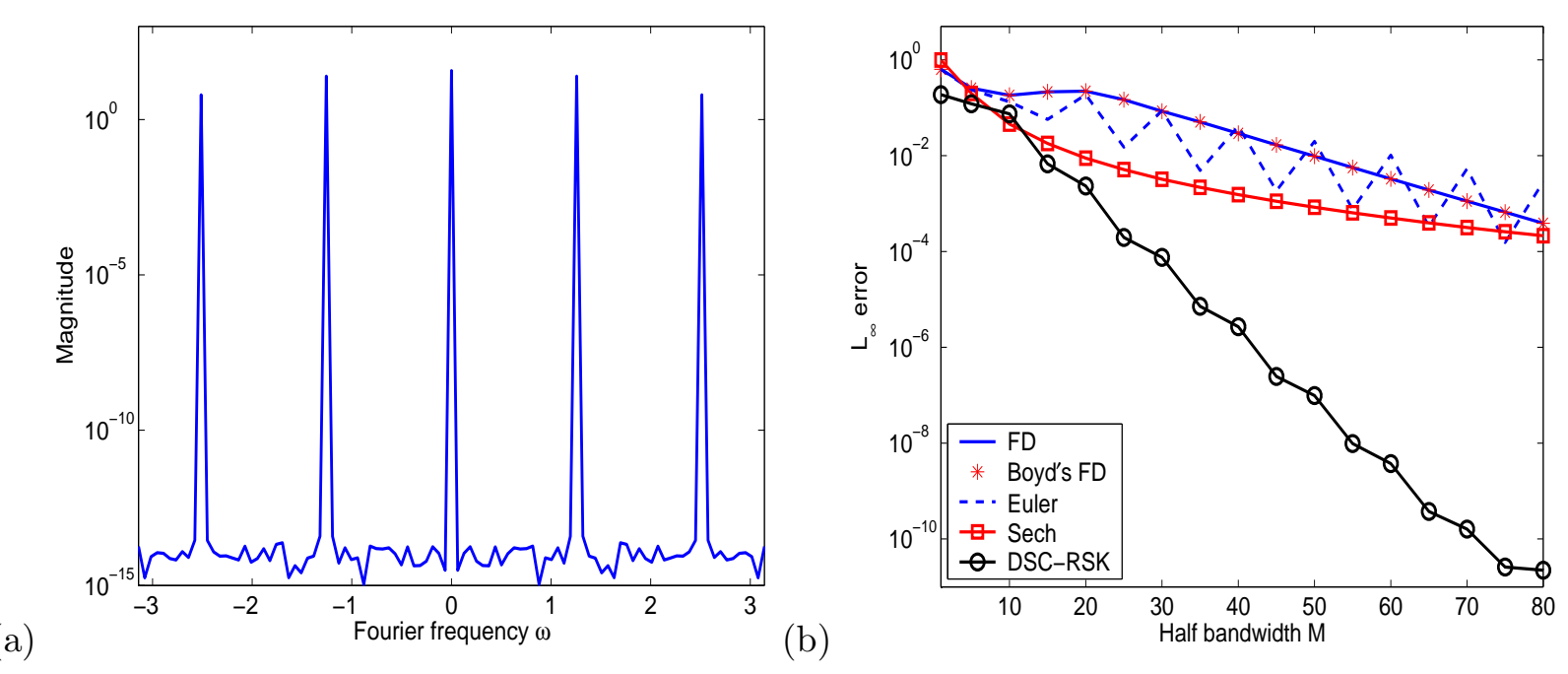

Figure 11: The unsteady hyperbolic equation with a few wavenumbers. (a) Fourier frequency response of $u(0, x)$; (b) Numerical errors.

the Sech method, a near optimal parameter $D=0.36$ is used. In the DSC-RSK method, we choose $(M, r)=(1,1.9),(5,2.4),(10,4.1),(15,5.3),(20,5.9),(25,6.7),(30,7.2),(35,7.8),(40,8.2),(45,8.8)$, $(50,9.2),(55,9.7),(60,10.0),(65,10.5),(70,10.8),(75,11.4)$, and $(80,11.5)$.

The $L_{\infty}$ errors are depicted in Fig. 11(b). Boyd's FD method yields the same results as the FD method. It is clear that for this highly oscillatory solution, the DSC-RSK method is the best for all $M$ values examined, including small stencils, like $M=1,5$ and 10 . When $M>75$, the DSCRSK error does not decay any more, because the accuracy limit of the RK4 temporal integration is reached. The accuracies of other approaches are far away from the limit. It is seen that the DSC-RSK method is over $10^{6}$ times more accurate than all other approaches examined for some large $M$.

\subsection{Unsteady hyperbolic equation with a wave comb}

We next consider Eq. (23) with a wide-band frequency solution

$$
u(t, x)=\sum_{j=1}^{k / 2} \sin \left(2 j \pi\left(x-t^{3} / 3\right)\right) .
$$

Fig. 12 (a) depicts the Fourier frequency response, which has a wide range of wavenumbers. To examine "Proof"'s claim about the FD method, we have chosen the amplitude in small wavenumbers to be as large as those in large wavenumbers. 

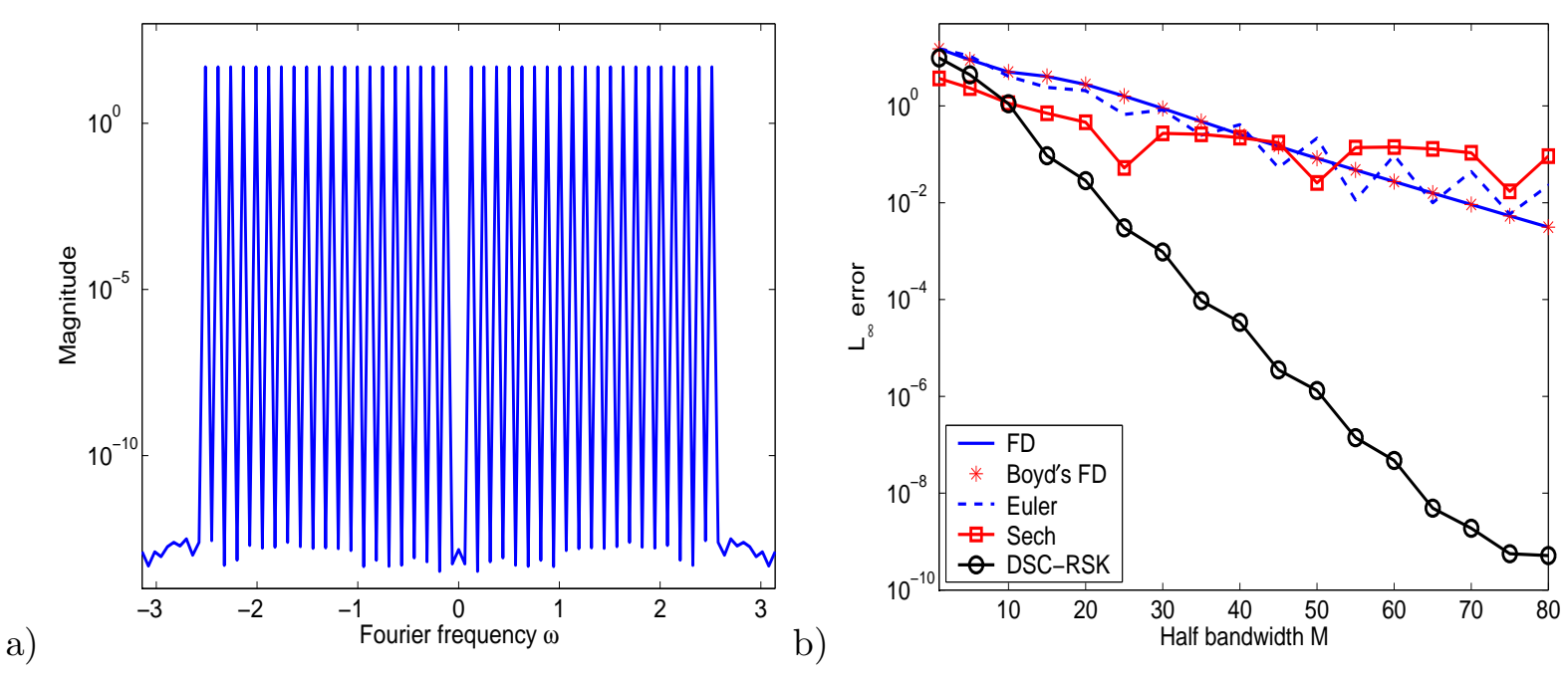

Figure 12: The unsteady hyperbolic equation with a wave comb. (a) Fourier frequency response; (b) Numerical errors.

Here we choose $N=101, k=40$, and $\Delta t=5 \times 10^{-5}$ in our computation. The free parameters $D$ and $r$ remain the same as in the last case. The results at $t=1$ are shown in Fig. 12 (b). Although none of the methods performs as well as in the previous case, the Sech method has the most dramatical accuracy reduction. Its error has increased 1000 times, indicating its sensitivity to the frequency distribution.

The DSC-RSK method keeps its edge over all other methods examined - it is up to a million times more accurate than the FD and Sech methods at large stencils. In fact, it outperforms the FD method over all stencil widths examined.

\subsection{Two dimensional Navier-Stokes equation}

We next consider the two dimensional (2D) Navier-Stokes equation in its primitive variable, describing an incompressible fluid flow

$$
\begin{aligned}
\frac{\partial \mathbf{u}}{\partial t}+\mathbf{u} \cdot \nabla \mathbf{u} & =-\nabla p+\frac{1}{\operatorname{Re}} \nabla^{2} \mathbf{u}, \\
\nabla \cdot \mathbf{u} & =0
\end{aligned}
$$


where $\mathbf{u}=(u, v)$ is the velocity vector, $p$ is the pressure and Re is the Reynolds number. In a $2 \mathrm{D}$ square domain $[0,2 \pi] \times[0,2 \pi]$ with periodic boundary conditions, the initial values are taken as

$$
\begin{aligned}
& u(0, x, y)=-\cos (k x) \sin (k y), \\
& v(0, x, y)=\sin (k x) \cos (k y) .
\end{aligned}
$$

The analytical solution is

$$
\begin{aligned}
u(t, x, y) & =-\cos (k x) \sin (k y) \exp \left(-2 k^{2} t / \mathrm{Re}\right), \\
v(t, x, y) & =\sin (k x) \cos (k y) \exp \left(-2 k^{2} t / \mathrm{Re}\right), \\
p(t, x, y) & =-\frac{1}{4}[\cos (2 k x)+\cos (2 k y)] \exp \left(-4 k^{2} t / \mathrm{Re}\right) .
\end{aligned}
$$

The present study has two interesting points in comparing with the previous studies, i.e., it is multi-dimensional and it involves nonlinearity. We adopt the Adams-Bashforth-Crank-Nicolson $(\mathrm{ABCN})$ scheme for the time discretization and treatment of pressure

$$
\begin{aligned}
\frac{1}{2 \operatorname{Re}} \nabla^{2} \mathbf{u}^{n+1}-\frac{1}{\Delta t} \mathbf{u}^{n+1} & =\nabla p^{n+1 / 2}+\mathbf{S}^{n} \\
\nabla^{2} p^{n+1 / 2}+\nabla \cdot \mathbf{S}^{n} & =0
\end{aligned}
$$

where the source vector $\mathbf{S}^{n}$ is given by

$$
\mathbf{S}^{n}=-\frac{\mathbf{u}^{n}}{\Delta t}+\frac{3\left(\mathbf{u}^{n} \cdot \nabla\right) \mathbf{u}^{n}-\left(\mathbf{u}^{n-1} \cdot \nabla\right) \mathbf{u}^{n-1}}{2}-\frac{1}{2 \operatorname{Re}} \nabla^{2} \mathbf{u}^{n}
$$

We consider a high frequency problem with $k=10$ and $\mathrm{Re}=100$. By using 51 nodes along each direction, the grid density for solving the Poisson equation of the pressure is PPW=2.5, while that for the velocity field is $\mathrm{PPW}=5$. In order to compare spatial discretization errors, a sufficiently small time increment, $\Delta t=1.0 \times 10^{-5}$, is used. For the Sech method, a near optimal $D=0.18$ is used. For the DSC-RSK method, we choose $(M, r)=(1,0.8),(5,1.7),(10,4.6),(15,5.4),(20,6.1)$, $(25,6.8),(30,7.3),(35,7.9),(40,8.3),(45,8.8)$, and $(50,9.3)$. The $L_{\infty}$ errors in $u$ after 1000 time steps are depicted in Fig. 13, It is clear that the DSC-RSK method is more accurate than other approaches for this high frequency problem when $M>25$. At $M=50$, the DSC-RSK method is over a thousand times more accurate than the Sech and FD methods.

\subsection{Quantum eigenvalue problem}

Finally, we consider a quantum eigenvalue problem

$$
\left[-\frac{1}{2} \frac{d^{2}}{d x^{2}}+V(x)\right] \Phi_{n}=E_{n} \Phi_{n}
$$




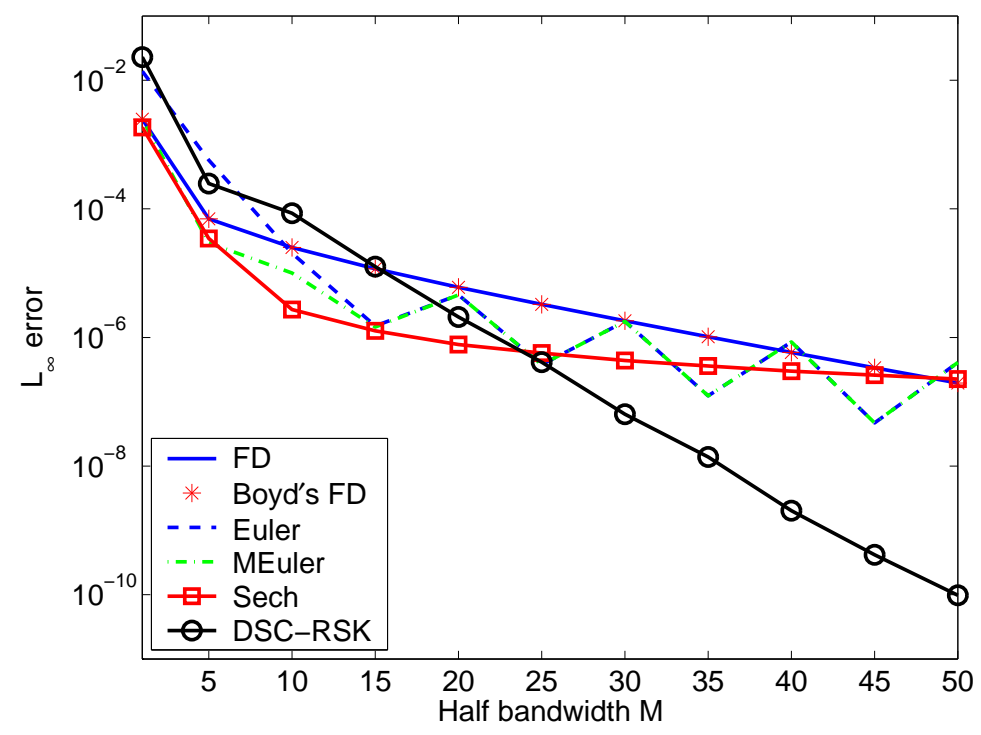

Figure 13: Numerical errors of the Navier-Stokes equation.

with harmonic oscillator potential $V(x)=x^{2} / 2$. The eigenfunctions $\Phi_{n}$ are the standard Hermite function series. The eigenvalues are $E_{n}=n+\frac{1}{2}$, where $n=0,1,2, \cdots$.

Table 3: Numerical errors in the quantum eigenvalue analysis with $M=50$ and $N=51$.

\begin{tabular}{llllllll}
\hline \hline Mode & Sinc & DSC-RSK & FD & Boyd's FD & Euler & MEuler & Sech \\
\hline 0 & $1.40(-14)$ & $9.10(-15)$ & $1.38(-13)$ & $7.17(-13)$ & $2.67(-15)$ & $2.67(-15)$ & $3.46(-14)$ \\
1 & $1.48(-14)$ & $3.85(-15)$ & $3.75(-14)$ & $2.23(-13)$ & $4.00(-15)$ & $4.00(-15)$ & $2.75(-14)$ \\
2 & $5.33(-15)$ & $5.33(-16)$ & $2.56(-14)$ & $1.18(-13)$ & $5.51(-15)$ & $5.51(-15)$ & $3.30(-14)$ \\
3 & $3.04(-15)$ & $1.27(-16)$ & $1.28(-14)$ & $7.74(-14)$ & $7.99(-15)$ & $7.99(-15)$ & $4.15(-14)$ \\
4 & $5.92(-16)$ & $5.92(-16)$ & $1.12(-14)$ & $5.51(-14)$ & $7.11(-15)$ & $7.11(-15)$ & $5.01(-14)$ \\
5 & $9.69(-16)$ & $4.84(-16)$ & $8.07(-15)$ & $4.00(-14)$ & $6.94(-15)$ & $6.94(-15)$ & $1.26(-14)$ \\
6 & $2.73(-16)$ & $1.64(-15)$ & $4.24(-15)$ & $3.07(-14)$ & $7.52(-15)$ & $7.52(-15)$ & $9.34(-13)$ \\
7 & $1.18(-15)$ & $4.74(-16)$ & $6.99(-15)$ & $2.21(-14)$ & $5.45(-15)$ & $5.45(-15)$ & $1.04(-11)$ \\
8 & $8.36(-16)$ & $2.09(-16)$ & $1.94(-14)$ & $4.60(-15)$ & $2.09(-15)$ & $2.09(-15)$ & $9.17(-11)$ \\
9 & $7.48(-16)$ & 0.00 & $1.01(-13)$ & $8.23(-14)$ & $2.43(-15)$ & $2.43(-15)$ & $7.03(-10)$ \\
10 & $5.07(-16)$ & 0.00 & $5.60(-13)$ & $5.45(-13)$ & $1.51(-13)$ & $1.51(-13)$ & $4.76(-9)$ \\
11 & $4.63(-16)$ & $1.54(-16)$ & $2.87(-12)$ & $2.86(-12)$ & $7.81(-13)$ & $7.81(-13)$ & $2.86(-8)$
\end{tabular}




\begin{tabular}{|c|c|c|c|c|c|c|c|}
\hline 12 & $7.11(-16)$ & $4.26(-16)$ & $1.35(-11)$ & $1.35(-11)$ & $1.58(-12)$ & $1.58(-12)$ & $1.53(-7)$ \\
\hline 13 & $1.32(-16)$ & $2.63(-16)$ & $5.83(-11)$ & $5.83(-11)$ & $2.09(-12)$ & $2.09(-12)$ & $7.37(-7)$ \\
\hline 14 & $3.68(-16)$ & $1.22(-16)$ & $2.33(-10)$ & $2.33(-10)$ & $2.31(-11)$ & $2.31(-11)$ & $3.18(-6)$ \\
\hline 15 & $1.38(-15)$ & $1.15(-15)$ & $8.68(-10)$ & $8.68(-10)$ & $6.40(-11)$ & $6.40(-11)$ & $1.23(-5)$ \\
\hline 16 & $6.46(-16)$ & $1.29(-15)$ & $3.01(-9)$ & $3.01(-9)$ & $3.65(-11)$ & $3.65(-11)$ & $4.31(-5)$ \\
\hline 17 & $7.31(-14)$ & $7.31(-14)$ & $9.79(-9)$ & $9.79(-9)$ & $3.23(-10)$ & $3.23(-10)$ & $1.35(-4)$ \\
\hline 18 & $5.57(-14)$ & $6.32(-14)$ & $2.99(-8)$ & $2.99(-8)$ & $1.19(-9)$ & $1.19(-9)$ & $3.78(-4)$ \\
\hline $1:$ & $2.87(-12)$ & $2.88(-12)$ & $8.61(-8)$ & $8.61(-8)$ & $1.54(-9)$ & $1.54(-9)$ & $9.48(-4)$ \\
\hline 20 & $1.78(-12)$ & $1.98(-12)$ & $2.34(-7)$ & $2.34(-7)$ & $1.96(-9)$ & $1.96(-9)$ & $2.12(-3)$ \\
\hline 91 & $8.66(-11)$ & $8.70(-11)$ & $6.04(-7)$ & $6.04(-7)$ & $1.24(-8)$ & $1.24(-8)$ & $4.26(-3)$ \\
\hline 22 & $4.42(-11)$ & $5.04(-11)$ & $1.48(-6)$ & $1.48(-6)$ & $2.17(-8)$ & $2.17(-8)$ & $7.71(-3)$ \\
\hline 23 & $1.99(-9)$ & $1.99(-9)$ & $3.45(-6)$ & $3.45(-6)$ & $3.79(-9)$ & $3.79(-9)$ & $1.27(-2)$ \\
\hline 2 & $7.87(-10)$ & $9.00(-10)$ & $7.67(-6)$ & $7.67(-6)$ & $7.55(-8)$ & $7.55(-8)$ & $1.94(-2)$ \\
\hline 25 & $3.57(-8)$ & $3.59(-8)$ & $1.63(-5)$ & $1.63(-5)$ & $1.40(-7)$ & $1.40(-7)$ & $2.78(-2)$ \\
\hline 26 & $1.12(-8)$ & $1.33(-8)$ & $3.33(-5)$ & $3.33(-5)$ & $1.23(-7)$ & $1.23(-7)$ & $3.77(-2)$ \\
\hline 27 & $4.93(-7)$ & $4.94(-7)$ & $6.44(-5)$ & $6.44(-5)$ & $7.59(-7)$ & $7.59(-7)$ & $4.90(-2)$ \\
\hline 28 & $1.05(-7)$ & $1.29(-7)$ & $1.23(-4)$ & $1.23(-4)$ & $1.08(-6)$ & $1.08(-6)$ & $6.16(-2)$ \\
\hline 29 & $5.34(-6)$ & $5.36(-6)$ & $2.15(-4)$ & $2.15(-4)$ & $6.43(-6)$ & $6.43(-6)$ & $7.52(-2)$ \\
\hline 30 & $7.32(-7)$ & $1.01(-6)$ & $4.04(-4)$ & $4.04(-4)$ & $2.05(-8)$ & $2.05(-8)$ & $8.98(-2)$ \\
\hline 31 & $4.40(-5)$ & $4.41(-5)$ & $6.01(-4)$ & $6.01(-4)$ & $4.02(-5)$ & $4.02(-5)$ & $1.05(-1)$ \\
\hline 32 & $7.74(-7)$ & $2.83(-6)$ & $1.24(-3)$ & $1.24(-3)$ & $2.88(-6)$ & $2.88(-6)$ & $1.21(-1)$ \\
\hline 33 & $2.81(-4)$ & $2.82(-4)$ & $1.33(-3)$ & $1.33(-3)$ & $2.81(-4)$ & $2.81(-4)$ & $1.38(-1)$ \\
\hline 34 & $2.87(-5)$ & $1.35(-5)$ & $3.86(-3)$ & $3.86(-3)$ & $2.54(-5)$ & $2.54(-5)$ & $1.56(-1)$ \\
\hline 35 & $1.33(-3)$ & $1.33(-3)$ & $1.93(-3)$ & $1.93(-3)$ & $1.34(-3)$ & $1.34(-3)$ & $1.74(-1)$ \\
\hline 36 & $3.82(-4)$ & $3.07(-4)$ & $1.16(-2)$ & $1.16(-2)$ & $3.62(-4)$ & $3.62(-4)$ & $1.92(-1)$ \\
\hline 37 & $4.83(-3)$ & $4.84(-3)$ & $4.36(-4)$ & $4.36(-4)$ & $4.83(-3)$ & $4.83(-3)$ & $2.11(-1)$ \\
\hline 38 & $1.48(-3)$ & $1.19(-3)$ & $2.04(-2)$ & $2.04(-2)$ & $1.54(-3)$ & $1.54(-3)$ & $2.31(-1)$ \\
\hline 39 & $1.34(-2)$ & $1.34(-2)$ & $6.66(-3)$ & $6.66(-3)$ & $1.33(-2)$ & $1.33(-2)$ & $2.50(-1)$ \\
\hline 40 & $2.95(-4)$ & $1.68(-4)$ & $1.71(-2)$ & $1.71(-2)$ & $1.86(-4)$ & $1.86(-4)$ & $2.70(-1)$ \\
\hline 41 & $3.06(-2)$ & $3.06(-2)$ & $2.29(-2)$ & $2.29(-2)$ & $3.07(-2)$ & $3.07(-2)$ & $2.91(-1)$ \\
\hline 42 & $1.19(-2)$ & $1.24(-2)$ & $1.05(-3)$ & $1.05(-3)$ & $1.19(-2)$ & $1.19(-2)$ & $3.11(-1)$ \\
\hline
\end{tabular}




\begin{tabular}{llllllll}
43 & $5.93(-2)$ & $5.93(-2)$ & $5.13(-2)$ & $5.13(-2)$ & $5.95(-2)$ & $5.95(-2)$ & $3.32(-1)$ \\
44 & $3.82(-2)$ & $3.86(-2)$ & $2.77(-2)$ & $2.77(-2)$ & $3.81(-2)$ & $3.81(-2)$ & $3.53(-1)$ \\
45 & $1.05(-1)$ & $1.06(-1)$ & $9.61(-2)$ & $9.61(-2)$ & $1.06(-1)$ & $1.06(-1)$ & $3.76(-1)$ \\
46 & $8.35(-2)$ & $8.39(-2)$ & $7.26(-2)$ & $7.26(-2)$ & $8.30(-2)$ & $8.30(-2)$ & $3.91(-1)$ \\
47 & $1.76(-1)$ & $1.76(-1)$ & $1.67(-1)$ & $1.67(-1)$ & $1.76(-1)$ & $1.76(-1)$ & $4.31(-1)$ \\
48 & $1.53(-1)$ & $1.53(-1)$ & $1.43(-1)$ & $1.43(-1)$ & $1.52(-1)$ & $1.52(-1)$ & $4.21(-1)$ \\
49 & $3.14(-1)$ & $3.14(-1)$ & $2.93(-1)$ & $2.93(-1)$ & $3.15(-1)$ & $3.15(-1)$ & $5.49(-1)$ \\
50 & $2.89(-1)$ & $2.90(-1)$ & $2.68(-1)$ & $2.68(-1)$ & $2.88(-1)$ & $2.88(-1)$ & $5.25(-1)$ \\
\hline \hline
\end{tabular}

Table 4: Numerical errors in the quantum eigenvalue analysis with $M=200$ and $N=201$.

\begin{tabular}{lllllll}
\hline \hline Mode & Sinc & DSC-RSK & FD & Euler & MEuler & Sech \\
\hline 0 & $8.49(-13)$ & $7.03(-13)$ & $8.70(-13)$ & 1.00 & 1.00 & $1.33(-13)$ \\
4 & $2.11(-14)$ & $6.12(-15)$ & $7.80(-14)$ & $9.86(-1)$ & $9.86(-1)$ & $1.42(-14)$ \\
8 & $2.28(-14)$ & $6.48(-15)$ & $9.57(-14)$ & $9.71(-1)$ & $9.71(-1)$ & $1.02(-14)$ \\
12 & $1.21(-14)$ & $1.02(-14)$ & $5.74(-14)$ & $9.55(-1)$ & $9.55(-1)$ & $9.66(-14)$ \\
16 & $3.66(-15)$ & $4.52(-15)$ & $5.23(-14)$ & $9.40(-1)$ & $9.40(-1)$ & $1.07(-13)$ \\
20 & $3.81(-15)$ & $9.70(-15)$ & $4.75(-14)$ & $9.24(-1)$ & $9.24(-1)$ & $1.52(-13)$ \\
24 & $3.48(-15)$ & $3.04(-15)$ & $4.21(-14)$ & $9.09(-1)$ & $9.09(-1)$ & $1.84(-13)$ \\
28 & $3.74(-16)$ & $4.24(-15)$ & $3.54(-14)$ & $8.93(-1)$ & $8.93(-1)$ & $1.36(-13)$ \\
32 & $4.15(-15)$ & $3.94(-15)$ & $3.24(-14)$ & $8.78(-1)$ & $8.78(-1)$ & $1.24(-13)$ \\
36 & $2.53(-15)$ & $2.92(-15)$ & $2.94(-14)$ & $8.63(-1)$ & $8.63(-1)$ & $8.10(-14)$ \\
40 & $7.02(-16)$ & $1.75(-15)$ & $2.72(-14)$ & $8.47(-1)$ & $8.47(-1)$ & $2.25(-13)$ \\
44 & $4.79(-16)$ & $4.79(-16)$ & $2.44(-14)$ & $8.32(-1)$ & $8.32(-1)$ & $2.32(-13)$ \\
48 & $1.46(-16)$ & $1.03(-15)$ & $2.27(-14)$ & $8.16(-1)$ & $8.16(-1)$ & $5.51(-13)$ \\
52 & 0.00 & $1.35(-16)$ & $2.18(-14)$ & $8.01(-1)$ & $8.01(-1)$ & $1.18(-12)$ \\
56 & $1.26(-16)$ & $2.51(-16)$ & $1.96(-14)$ & $7.85(-1)$ & $7.85(-1)$ & $1.53(-12)$ \\
60 & $3.52(-16)$ & $2.35(-16)$ & $1.77(-14)$ & $7.70(-1)$ & $7.70(-1)$ & $1.72(-12)$ \\
64 & 0.00 & $8.81(-16)$ & $1.74(-14)$ & $7.54(-1)$ & $7.54(-1)$ & $1.06(-12)$ \\
68 & $2.07(-16)$ & 0.00 & $1.53(-14)$ & $7.39(-1)$ & $7.39(-1)$ & $9.25(-14)$
\end{tabular}




\begin{tabular}{|c|c|c|c|c|c|c|}
\hline & 6) & 6) & 1) & $.23(-1)$ & $3(-1)$ & $(-12$ \\
\hline & $57(-16)$ & $3.72(-16)$ & $1.34(-14)$ & $7.08(-1)$ & $7.08(-1)$ & $1.45(-12)$ \\
\hline & 00 & & $29(-14)$ & $6.92(-1)$ & $92(-1)$ & 10 \\
\hline & & $6(-$ & 1) & $6.77(-1)$ & $77(-1)$ & 10 \\
\hline & 00 & 16) & $19(-14)$ & $6.61(-1)$ & $61(-1)$ & $1.43(-12)$ \\
\hline & $3.07(-16)$ & $3.07(-16)$ & $.69(-14)$ & $6.46(-1)$ & $6.46(-1)$ & $3.58(-12)$ \\
\hline & $.95(-16)$ & 0.00 & $.41(-13)$ & $6.30(-1)$ & $30(-1)$ & $46(-12)$ \\
\hline & $66(-16)$ & $2.83(-16)$ & $12(-12)$ & $6.15(-1)$ & $5(-1)$ & $(-13)$ \\
\hline & $5.44(-16)$ & 0.00 & $82(-11)$ & $5.99(-1)$ & $5.99(-1)$ & $5.98(-12)$ \\
\hline & $6.55(-16)$ & 6551 & $3.11(-10)$ & $5.84(-1)$ & $5.84(-1)$ & $2.65(-11)$ \\
\hline & $32(-16)$ & 1.0 & ( & 1) & $3(-1)$ & -11 \\
\hline & $6.10(-16)$ & $1.22(-16)$ & 1 & $5.53(-1)$ & $.53(-1)$ & -11 \\
\hline & $2.19(-14)$ & & 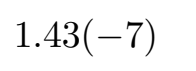 & $5.37(-1)$ & $7(-1)$ & 10 \\
\hline & $25(-12)$ & $1.26(-12)$ & $.88(-7)$ & $5.22(-1)$ & $5.22(-1)$ & $2.11(-10$ \\
\hline & $5.35(-11)$ & $5.39(-11)$ & $371(6)$ & $5.06(-1)$ & $5.06(-1)$ & $160(-10$ \\
\hline & $1.70(-9)$ & $1.72(-9)$ & $1.51(-5)$ & $4.91(-1)$ & $4.91(-1)$ & $1.45(-9)$ \\
\hline & $3.94(-8)$ & $3.99(-8)$ & $(-5)$ & $4.75(-1)$ & $4.75(-1)$ & $3.91(-8)$ \\
\hline & $47(-7)$ & $5 n(-7)$ & 101 & $60(-1)$ & $4.60(-1)$ & $.47(-7)$ \\
\hline & $14(-0)$ & $.00(1$ & 30 & $4.44(-1)$ & $4.44(-1)$ & $7.14(-6)$ \\
\hline & $4.66(-5)$ & & & 29( & $29(-1)$ & $4.66(-5)$ \\
\hline & $1.21(-4)$ & $1.39(-4)$ & $5.24(-3)$ & $4.13(-1)$ & $4.13(-1)$ & $1.21(-4)$ \\
\hline & $86(-5)$ & $1.60(-4)$ & 0.017 & $3.98(-1)$ & $3.98(-1)$ & $7.86(-5)$ \\
\hline & $1.97(-3)$ & $2.08(-3)$ & $.5 r-3$ & $3.82(-1)$ & $3.82(-1)$ & $1.97(-3)$ \\
\hline & $39(-3)$ & $.48(-3)$ & $.90(-$ & $3.67(-1)$ & $3.67(-1)$ & $8.39(-3)$ \\
\hline & $91(-2)$ & $1.92(-2)$ & 2) & $3.51(-1)$ & $3.51(-1)$ & $1.91(-2)$ \\
\hline & $3.39(-2)$ & $3.40(-2$ & $.12(-2)$ & $3.36(-1)$ & $3.36(-1)$ & $3.39(-2)$ \\
\hline & $5.31(-2)$ & (2) (2) & $0.00(-2)$ & $3.21(-1)$ & $3.21(-1)$ & $5.31(-2)$ \\
\hline & $7.74(-2)$ & $7.75(-2)$ & $7.48(-2)$ & $3.05(-1)$ & $3.05(-1)$ & $7.74(-2)$ \\
\hline & $.08(-1)$ & $1.08(-1)$ & $1.05(-1)$ & $2.89(-1)$ & $2.89(-1)$ & $1.08(-1)$ \\
\hline & $1.47(-1)$ & $1.47(-1)$ & $1.44(-1)$ & $2.74(-1)$ & $2.74(-1)$ & $1.47(-1)$ \\
\hline & $.99(-1)$ & $1.99(-1)$ & $1.96(-1)$ & $2.59(-1)$ & $2.59(-1)$ & $1.99(-1)$ \\
\hline
\end{tabular}




\begin{tabular}{lllllll}
196 & $2.74(-1)$ & $2.74(-1)$ & $2.69(-1)$ & $2.43(-1)$ & $2.43(-1)$ & $2.74(-1)$ \\
200 & $4.15(-1)$ & $4.15(-1)$ & $4.05(-1)$ & $2.28(-1)$ & $2.28(-1)$ & $4.15(-1)$ \\
\hline \hline
\end{tabular}

For the Sech method, a near optimal $D=0.28$ is used. In the DSC-RSK method, relatively large values of $r$ are chosen: $(M, r)=(50,35)$, and $(200,90)$. For $N=51$, and 201 , the computational domain is chosen as $x \in[-8.7,8.7]$, and [-17.6, 17.6], respectively.

This example is chosen to show the subtleness in numerical analysis. A method that is good for one problem might not be good for other problems. Detailed analysis is required for a given problem. In particular, we show that the analysis and claim in "Proof" about the Sinc pseudospectral method is invalid.

The relative errors in estimating eigenmodes for different $M$ with $N=M+1$ are given in Table 3 and Table 4. Since the $O(1 / x)$ decay in the Sinc kernel is outpaced by the Gaussian decay of the Hermite functions when $|x|$ is large, there is no need for sum acceleration. In fact, the Euleraccelerated sinc method (Euler) does not work as well as the Sinc does. Clearly, the Sinc method is the best for all $M$ and all eigenmodes. As it is known that when $r \rightarrow \infty$, the DSC-RSK method approaches to the Sinc method, a very large $r$ is chosen for each $M$, such that the DSC-RSK method results are very similar to those of the Sinc. Thus, overall, the DSC-RSK method performs better than the FD, Euler, MEuler, and Sech methods.

What comes as a surprise is the Sech method, which is able to deliver results of double precision accuracy - in contrast to the fact that it does not reach the accuracy of $10^{-7}$ in all other test cases. Overall, although it is not as accurate as the Sinc and DSC-RSK method, the Sech method performs better than the FD, Euler and MEuler methods.

When $M \geq 50$, the results of the Euler and MEuler methods are essentially the same. In particular, for $M=200$, they all yield wrong results.

\section{Analysis of "Proof"'s miscellaneous claims}

In this section, we further analyze "Proof"'s claims. For convenience, these claims will be presented in an itemized style.

- ["Proof"- Section 1] "It is impossible to review the work of this very prolific author in detail, but of the more than one hundred articles listed at http://www.math.msu.edu/wei/ most use the DSC or LDAF schemes." 
[ANALYSIS] The contrary is true: More than half of the articles do not use the DSC or LDAF schemes.

- ["Proof"- Section 1] "Since the finite difference weights are nearly Gaussian, one cannot escape the conclusion that the LDAF/DSC methods are really just high order finite difference methods in disguise!"

[ANALYSIS] There is no disguise about the relation between the DSC and high order finite differences in our work, as the relation has been spelled out many times in the DSC literature (e.g. in [23, 24]). It is surprising to see this accusation as the author was informed of the existence of these references in a comment on his draft. Note that all collocation schemes, including the Daubechies wavelet collocation method, can be cast into the finite difference form [11. However, the DSC method has also been formulated in the Galerkin form [17] for solving differential equations, which is not a high order finite difference method.

- ["Proof" - Section 1] "The LDAF/DSC is also a special case of Boyd's earlier theory of sumaccelerated pseudospectral methods: special in that the weighting function is a Gaussian." [AnALYsis] This is an unfounded claim. First, as a computational (discrete) realization of singular convolution kernels, including kernels of delta type, Abel type and Hilbert type [22], the DSC has little to do with Boyd's sum-accelerated method.

Second, the DSC-RSK approximation of derivatives does not fit into Boyd's sum-acceleration form $\frac{\mathrm{d} u}{\mathrm{~d} x} \approx \sum_{j=-M}^{M} w_{M j}^{\mathrm{DSC}} d_{j}^{(1), \text { sinc }}$ in general — there is an extra term, see Section 3. Only in a very special case, the on-grid differentiation for the first derivative, can the DSC-RSK method reduce to a form similar to Boyd's sum-accelerated pseudospectral methods. However, off-grid differentiations, which have been widely used in staggered grids in the previous DSC-RSK applications, cannot. More extra terms occur even for on-grid differentiations when higher order derivatives are considered.

Finally, the DSC-RSK method is constructed by accelerating the Whittaker-Shannon-Kotel'nikov sampling (interpolation) kernel.

- ["Proof"- Section 2] "Fourier analysis has been widely used to analyze difference formulas ever since this was popularized by von Neuman(n). The reason is that the Fourier basis function, $\exp ^{(i k x)}$, is an eigenfunction of both the differentiation operator and also of all 
possible difference formulas. This implies that the accuracy of difference formulas can be assessed - and improved - merely by comparing the eigenvalues."

[ANALYSIS]

First, it is inappropriate to state that "the accuracy of difference formulas can be assessed - and improved - merely by comparing the eigenvalues". In general, the performance of a difference formula for approximating a derivative of a function cannot be assessed by its performance on differentiating $\exp ^{(i k x)}$. The reason is the follows. In differentiating $\exp ^{(i k x)}$, the (discrete) Fourier method is exact and all difference formulas are less accurate. This does not justify the Fourier method to be a universal standard for judging a numerical method's performance of differentiation of a general function, because the performance always depends on the functional class, regularity, decay rate, etc. A simple counterexample to "Proof"'s logic is that in differentiating an $n$th order polynomial, an $n$th order FD method is exact, while the Fourier method is not.

Second, in solving differential equations, the superiority of differentiating $\exp ^{(i k x)}$ does not establish the superiority of a numerical method in general, since differential equations might involve functions of different mathematical properties, and be either nonlinear, or with variable coefficients, or with other complex non-differentiating terms. For example, the Hermite spectral method does not give an exact result for the differentiation of $\exp ^{(i k x)}$ as the Fourier method does. However, it can give the exact solution to the quantum Harmonic oscillator problem, see Eq. (31), whereas, the Fourier method can only provide approximations to this solution. Therefore, rigorous numerical analysis should always be done case by case, with detailed consideration of functional class and various constraints.

- ["Proof"- Section 2] "In practice, the requirement that $u(x)$ decays exponentially for $|x| \rightarrow \infty$ implies that the grid can be truncated to some large-but-finite span; the derivative sums are then truncated to summations over the entire truncated grid."

[AnALYsis] The Fourier basis function $u(x)=e^{i k x}$ is widely used in practice as well as in "Proof", and does not decays exponentially for $|x| \rightarrow \infty$.

- ["Proof"- Section 3] "However, the problem of summing slowly convergent series is an ancient one. A broad collection of schemes, known variously as "summability", "sequence acceleration" or "sum-acceleration" methods have been developed. Boyd [5] was the first to 
apply such ideas to pseudospectral series to invent the form of nonstandard differences called "sum-accelerated pseudospectral"."

[ANALYSIS] The general idea of accelerating the Whittaker-Shannon-Kotel'nikov sampling, i.e., the sinc pseudospectral series, with a weight function was introduced as early as 1919 by M. Theis [21]. Campbell introduced a weight of the form $w(x)=\frac{\int_{-1}^{1} \exp \left[\left(1-t^{2}\right)^{-1}-i t x\right] d t}{\int_{-1}^{1} \exp \left[\left(1-t^{2}\right)^{-1}-i t x\right] d t}$ to the sinc pseudospectral series in 1968 [7].

- ["Proof"- Section 3] "The sinc differentiation eigenvalue for the first derivative is the usual sine series $\cdots$. The first option to obtain a sparse stencil is to simply truncate the infinite series at some upper limit $n$. This is a really bad idea because the error in the series, truncated after $n$ terms, is $O(1 / n)$, and therefore unacceptably large."

[ANALYSIS] Note that "the sinc differentiation eigenvalue for the first derivative" refers to the result of differentiating $e^{(i k x)}$ with the Sinc method. The truncation error of the sinc pseudospectral method depends not only on the decay rate of the differentiation kernel, but also on the decay rate of the function being differentiated. This partially explains why the performance in differentiating $e^{(i k x)}$ does not translate directly into the performance in differentiating other functions. In Section 4.11 we show that for a quantum eigenvalue problem whose solution is of Schwartz class, the sinc collocation outperforms the Boyd's Euler-accelerated sinc (Euler) and spectrally weighted least square differences (Sech) by many orders of magnitude, see Tables 3 and 4 .

- ["Proof"- Section 5] "The shaded region shows that when $a<a_{\mathrm{FD}}$, the finite difference error is worse than that for the Gaussian weighting but only for $K>\pi / 2$."

[AnALYSIS] Section 4.1 provides a counterexample. Ironically, many more counterexamples appear in Fig. 2 of "Proof".

- ["Proof"- Section 5] "Given that the Fourier spectra of smooth functions fall off exponentially as $|K|$ increases, even small errors near $K=0$ are intolerable."

[ANALYSIS] There is a simple counterexample: The Fourier spectra of smooth functions, such as the $C^{\infty}$ function of $e^{-x} \sin (x)$, do not fall off exponentially as $|K|$ increases.

- ["Proof"- SeCtion 5] "Why are finite differences so superior to DSC, given that both employ weights that are close-to-Gaussian? The answer is that the finite difference weights 
are tuned to give maximum accuracy in the limit $K \rightarrow 0 . "$

[ANALYSIS] It is well-known that high order finite difference weights are exact in differentiating polynomials of the same order or less, which have a wide Fourier spectral distribution beyond the component of $K \rightarrow 0$.

- ["Proof"- Section 7] "How can the DSC/LDAF algorithms be salvaged? One could of course try a different sum-acceleration weighting from the Gaussian. However, this is merely to explore various instances of the sum-acceleration methods of [5]. "

[ANALYSIS]

The many examples given here give the reader sufficient information to judge the validity of this claim.

\section{Concluding remarks}

This paper examines the validity of claims made in Boyd's paper, "A proof that the discrete singular convolution (DSC)/Lagrange-distributed approximation function (LDAF) method is inferior to high order finite differences" [6], which is referred to as "Proof". A wide variety of test problems are employed to compare six different numerical methods, including the discrete singular convolution with the regularized Shannon kernel (DSC-RSK), the standard finite difference (FD), Boyd's spectrally-weighted difference with the sech weight (Sech) [5], Boyd's finite difference (Boyd's FD) [5], Boyd's Euler-accelerated sinc algorithm (Euler) [4, 5], and Boyd's modified Euler-accelerated sinc algorithm (MEuler) [4, 5]. These methods are employed in our comparisons because "Proof" has placed great emphasis of them, including many detailed expressions and/or numerical procedures, and because they are related to some of "Proof"'s claims. Some of the test problems, such as differentiating $e^{i k x}$ and a boundary value problem, are the designated tests used in either "Proof" or Boyd's earlier literature [5]. In numerous examples, the DSC-RSK method outperforms all the other five methods by factors of multiple orders of magnitude. In particular, for many problems with large wavenumbers or a wide range of wavenumbers (including large amplitudes in small wavenumbers), the DSC-RSK method is up to a million times more accurate than any of the other five methods mentioned.

Although "Proof" presents error expressions for both the FD and DSC-RSK methods for differentiating the Fourier basis function, its claims are not directly supported by these error expressions, 
and the claim about the superiority of the FD method over the DSC-RSK method is based on numerical experiments with a limited set of parameters, and on some informal arguments. Moreover, "Proof"'s claim about the superiority of spectrally-weighted differences over the DSC-RSK method is not supported by any analysis.

While we demonstrate that the DSC-RSK method outperforms the FD method for differentiating $e^{(i k x)}$ with fairly small wavenumbers, it may be the case that the FD method is superior with very small wavenumbers. In fact, the DSC-RSK method's less accurate performance in differentiating functions with solely very small wavenumbers is nothing new to us. It is for this reason that the DSC-RSK method was not proposed as another finite difference scheme. Instead, it was proposed as a local spectral method, to be used for problems that are difficult for both low order methods and global spectral methods. High frequency problems in flows, structural vibrations, and electromagnetic wave scattering and propagation are typical examples [3, 17, 25, 26, 27, 28]. The DSC algorithm had hardly been used in small stencils in its applications, except for a couple of cases in complying with referee's requests. Nevertheless, for certain problems, it could outperform the FD method with small stencils as shown in Section 4.1 and many other examples. The analysis of spectral convergence of the DSC-RSK method was given for certain class of functions in [19].

One may expect that for some physical problems where functions have Fourier spectra with amplitudes decaying exponentially, the ability to accurately approximate large wavenumbers is not important. In Section 4.2 we present an example in which the function has exponentially decaying amplitudes in wavenumbers $k$, i.e., amplitudes in large wavenumbers are exponentially small comparing to those in small wavenumbers. Contrary to "Proof"'s claims, the DSC-RSK method outperforms the FD method over all the stencils examined, and is up to $10^{6}$ times more accurate than the FD method and Boyd's spectrally weighted difference, the Sech method, with some large stencils. This example indicates that for six numerical methods examined, the dominant errors in derivatives originate from large wavenumbers. Therefore, a method that is not only accurate for low wavenumbers, but also able to deliver high accuracy for large wavenumbers, will be more useful for many physical problems whose Fourier spectra decay exponentially.

Indeed, many problems examined in this work involve considerable amplitudes in either large wavenumbers, or a wide range of wavenumbers which include both small and large wavenumbers. These problems are not suitable for low order methods - they require high order methods with relatively large stencils to achieve highly accurate results. In designing counterexamples, emphasis 
was not given to physical origins, partially because the claims of "Proof" have very little to do with applications, and partially because the DSC-RSK method has been applied to many practical problems in the past, in particular, to problems involving large wavenumbers [3, 17, 25, 26, 27, 28. The importance of large wavenumbers, or 'short waves', cannot be overemphasized in scientific and engineering applications. At the beginning of the new millennium, Zienkiewicz [29] listed the problem of short waves in acoustics, electromagnetics or surface wave applications as one of two unsolved computational problems. Babuška and coworkers [1, 2, 9, 14, 15] devoted much effort to constructing advanced numerical methods for high frequency waves in the Helmholtz equation. Engquist and his co-workers [10] proposed the segment projection method for the propagation of high-frequency waves in waveguides. Shu and Osher [20] designed highly oscillatory problems in hyperbolic conservation laws to validate high-order shock-capturing methods. In space science and aerospace engineering, it is pertinent to quote Langley and Bardell [16] from their review paper: "...the prediction of medium to high frequency vibration levels is a particularly difficult task. ...there is no single technique which can be applied with confidence to all types of aerospace structures. Furthermore, there are certain problems of pressing practical concern for which it is not possible at present to make a reliable design prediction of high frequency vibration levels - the prediction of on-orbit micro-vibration levels in satellite structures is arguably a problem of this type".

Like Boyd's Sech method, the DSC-RSK method has a free parameter $r$. Since "Proof"'s claim was very strong, specifically including all $a<a_{\mathrm{FD}}$ (i.e., all $r>\frac{1}{\sqrt{2} a_{\mathrm{FD}}}$, see "Proof"'s Abstract), we have chosen near optimal $r$ values in this work. In practice, $r$ values can be optimized according to specific applications. For a given stencil, there is a quite wide range of $r$ values that deliver very good results. Hence, the DSC-RSK method is relatively robust. Although it takes some experience and understanding to choose a near optimal $r$ value, there is no need to know the exact solution a prior. If one wishes to obtain a near optimal $r$ value, one can analyze the Fourier frequency response of the numerical solution. A near optimal $r$ value can then be obtained by elevating (or decreasing) the first choice of $r$ if the Fourier frequency response involves very high frequency components (or solely low frequency components).

The sole purpose of this paper is to analyze the validity of "Proof". Although the reported counterexamples are a very small fraction of counterexamples we know, we have no intention to claim the superiority of the DSC-RSK algorithm. Given the great diversity of problems with different physical origins, it is improper to claim that one method is superior to others in general 
without detailed analysis and comparison. However, for a given problem, one could show that some methods are more suitable than others. Case-by-case study is very important for validating new numerical methods. In view of the fact that detailed comparisons between the DSC-RSK method and many other numerical methods, including many other spectrally-weighted differences, have not been made anywhere, it is entirely possible to find another method that outperforms the DSC-RSK method for some examples studied in this work. It is also possible to come up with other examples for which the DSC-RSK method does not perform as well as the other five finite difference-type methods examined in this work. However, these possibilities do not affect the conclusion of the present paper, which demonstrates that the general statements in "Proof" are unfounded.

\section{Acknowledgments}

This work was supported in part by NSF Grant IIS-0430987.

\section{References}

[1] I. Babuška, Is the pollution effect of the FEM avoidable for the Helmholtz equation considering high wave numbers?, SIAM J. Numer. Anal., 34 (1997), pp. 2392.

[2] I. Babuška, T. Strouboulis, S.K. Gangaraj and C.S. Upadhyay, Pollution error in the h-version of the finite element method and the local quality of the recovered derivatives, Comput. Meth. Appl. Mech. Engng., 140 (1997), pp. 1-37.

[3] G. Bao, G.W. Wei, and S. Zhao, Numerical solution of the Helmholtz equation with high wave numbers, Int. J. Numer. Meth. Engng., 59 (2004), pp. 389-408.

[4] J.P. Boyd, Sum-accelerated pseudospectral methods: the Euler-accelerated sinc algorithm, Appl. Numer. Math., 7 (1991), pp. 287-296.

[5] J.P. Boyd, Sum-accelerated pseudospectral methods: finite differences and sech-weighted differences, Comput. Methods Appl. Mech. Engng., 116 (1994), pp. 1-11.

[6] J.P. Boyd, A proof that the discrete singular convolution (DSC)/Lagrange-distributed approximation function (LDAF) method is inferior to high order finite differences, J. Comput. Phys., 214 (2006), pp. 538-549. 
[7] L. Campbell, Sampling theorem for the Fourier transform of a distribution with bounded support, SIAM J. Applied Math., 16 (1968), pp. 626-636.

[8] W.J. Cody, Specfun - A portable Fortran package of special function routines and test drivers, ACM Transactions on Mathematical Software, 19 (1993), pp. 22-32.

[9] A. Deraemaeker, I. Babuška and P. Bouillard, Dispersion and pollution of the FEM solution for the Helmholtz equation in one, two and three dimensions, Int. J. Numer. Meth. Engng., 46 (1999), pp. 471-499.

[10] B. Engquist, Q. Runborg and A.K. Tornberg, High-frequency wave propagation by the segment projection method, J. Comput. Phys., 178 (2002), pp. 373-390.

[11] B. Fornberg, A Practical Guide to Pseudospectral Methods, Cambridge University Press, Cambridge, 1996.

[12] B. Fornberg, Calculation of weights in finite difference formulas, SIAM Rev., 40 (1998), pp. 685-691.

[13] D.K. Hoffman, N. Nayar, O.A. Sharafeddin and D.J. Kouri, Analytic banded approximation for the discretized free propagator, J. Phys. Chem-US, 95 (1991), pp. 8299-8305.

[14] F. Ihlenburg and I. Babuška, Finite-element solution of the Helmholtz-equation with high wavenumber. 1. The H-version of the FEM, Comput. Math. Appl., 30 (1995), pp. 9-37.

[15] F. Ihlenburg and I. Babuška, Finite element solution of the Helmholtz equation with high wave number. 2. The h-p version of the FEM, SIAM J. Numer. Aanal., 34 (1997), pp. 315-358.

[16] R.S. Langley and N.S. Bardell, A review of current analysis capabilities applicable to the high frequency vibration prediction of aerospace structures, The Aeronautical Journal, 102 (1998), pp. 287-297.

[17] C.W. Lim, Z.R. Li and G.W. Wei, DSC-Ritz method for the high frequency mode analysis of thick shallow shells, Int. J. Numer. Meth. Engng., 62 (2005), pp. 205-232.

[18] W.H. Press, S.A. Teukolsky, W.T. Vetterling and B.P. Flannery, Numerical recipes in Fortran: the art of scientific computing, Cambridge University Press, 1999. 
[19] L.W. Qian, On the regularized Whittaker-Kotel'nikov-Shannon sampling formula, Proc. Amer. Math. Soc., 131 (2003), pp. 1169-1176.

[20] C.W. Shu and S. Osher, Efficient implementation of essentially non-oscillatory shock-capturing schemes. 2., J. Comput. Phys., 83 (1989), pp. 32-78.

[21] M. Theis, Über eine interpolations formel von de la Vallée Poussin, Math. Z., 3 (1919), pp. 93-113.

[22] G.W. Wei, Discrete singular convolution for solution of the Fokker-Planck equations, J. Chem. Phys., 110 (1999), pp. 8930-8942.

[23] G.W. Wei, A unified approach for solving the Fokker-Planck equation, J. Phys. A, Math. Gen., 33 (2000), pp. 4935-4953.

[24] G.W. Wei, Y.B. Zhao and Y. Xiang, Discrete singular convolution and its application to the analysis of plates with internal supports. I Theory and algorithm, Int. J. Numer. Methods Engng., 55 (2002), pp. 913-946.

[25] G.W. Wei, Y.B. Zhao, and Y. Xiang, A novel approach for the analysis of high frequency vibrations, J. Sound Vibration, 257 (2002), pp. 207-246.

[26] Y.B. Zhao, G.W. Wei, and Y. Xiang, Discrete singular convolution for the prediction of high frequency vibration of plates, Int. J. Solids and Struct., 39 (2002), pp. 65-88.

[27] Y.C. Zhou and G.W. Wei, High-resolution conjugate filters for the simulation of flows, J. Comput. Phys., 189 (2003), pp. 150-179.

[28] Y.C. Zhou, Y. Gu and G.W. Wei, Shock-capturing with natural high frequency oscillations, Int. J. Numer. Methods Fluid, 41 (2003), pp. 1319-1338.

[29] O. C. Zienkiewicz, Achievements and some unsolved problems of the finite element method, Int. J. Numer. Meth. Engng., 47 (2000), pp. 9-28. 